\title{
Nonlinear Evolution Governed by Accretive Operators in Banach Spaces: Error Control and Applications
}

\author{
Ricardo H. Nochetto \\ Department of Mathematics and Institute for Physical Science \\ and Technology, University of Maryland * \\ Giuseppe Savaré \\ Dipartimento di Matematica, Università di Pavia, and \\ I.M.A.T.I., C.N.R. ${ }^{\dagger}$
}

\begin{abstract}
Nonlinear evolution equations governed by $m$-accretive operators in Banach spaces are discretized via the backward or forward Euler methods with variable stepsize. Computable a posteriori error estimates are derived in terms of the discrete solution and data, and shown to converge with optimal order $O(\sqrt{\tau})$. Applications to scalar conservation laws and degenerate parabolic equations (with or without hysteresis) in $L^{1}$, as well as to Hamilton-Jacobi equations in $C^{0}$ are given. The error analysis relies on a comparison principle, for the novel notion of relaxed solutions, which combines and simplifies techniques of Benilan and Kružkov. Our results provide a unified framework for existence, uniqueness and error analysis, and yield a new proof of the celebrated Crandall-Liggett error estimate.
\end{abstract}

\section{Introduction}

Let $\mathscr{B}$ be a Banach space with norm $\|\cdot\|$ and let $\mathfrak{F}$ be a (possibly multivalued) operator in $\mathscr{B}$ with domain $D(\mathfrak{F}) \subset \mathscr{B}$. Given an initial datum $u_{0}$ in the closure of $D(\mathfrak{F})$ and a forcing function $f \in L^{1}(0, T ; \mathscr{B})$, we analyze the approximation of the Cauchy problem

$$
\left\{\begin{array}{l}
u^{\prime}(t)+\mathfrak{F}(u(t)) \ni f(t) \\
u(0)=u_{0}
\end{array}\right.
$$

by a variable step implicit or explicit Euler method. More precisely, given

$$
\text { the partition } \mathcal{P}=\left\{t_{0}:=0<t_{1}<\ldots<t_{N-1}<t_{N}:=T\right\}
$$

* College Park, MD 20742, USA (E-mail: rhn@math.umd.edu). Partially supported by NSF Grants DMS-9971450 and DMS-0204670.

$\dagger$ Via Ferrata 1, 27100 Pavia, Italy (E-mail: savare@imati.cnr.it, Web: http://www.imati.cnr.it/ savare). Partially supported by M.I.U.R. Grants Cofin1999,2002 
of the time interval $[0, T]$ with variable step-size

$$
\tau_{n}:=t_{n}-t_{n-1}, \quad \tau:=\max _{n} \tau_{n}
$$

and given $U_{0} \in D(\mathfrak{F})$ and the sequence $\left\{F_{n}\right\}_{n=1}^{N} \subset \mathscr{B}$, we consider the sequence $\left\{U_{n}\right\}_{n=0}^{N}$ defined recursively by the Implicit Euler Scheme

$$
\frac{U_{n}-U_{n-1}}{\tau_{n}}+\mathfrak{F}\left(U_{n}\right) \ni F_{n}, \quad \forall 1 \leq n \leq N,
$$

or by the Explicit Euler Scheme

$$
\frac{U_{n}-U_{n-1}}{\tau_{n}}+\mathfrak{F}\left(U_{n-1}\right) \ni F_{n}, \quad \forall 1 \leq n \leq N .
$$

Observe that $\left(\mathrm{IS}_{1}\right)$ requires to solve at each step a problem of the type

$$
\text { given } w \in \mathscr{B} \quad \text { find } v \in D(\mathfrak{F}): \quad v+\lambda \mathfrak{F}(v) \ni w
$$

where $\lambda$ is one of the time steps, so that it is natural to suppose

$$
\text { the map } I+\lambda \mathfrak{F} \text { is surjective for } 0<\lambda \leq \tau \text {. }
$$

In order to guarantee solvability of an arbitrary number of iterations of $\left(\mathrm{ES}_{1}\right)$ and to avoid ambiguities we have to assume that

$$
D(\mathfrak{F})=\mathscr{B}, \quad \mathfrak{F} \quad \text { is single-valued. }
$$

In both cases, our crucial assumption relies on a contractivity assumption of the maps " $U_{n-1} \mapsto U_{n}$ " defined by $\left(\mathrm{IS}_{1}\right)$ or $\left(\mathrm{ES}_{1}\right)$ when $F_{n}=0$. In fact, for the implicit scheme $\left(\mathrm{IS}_{1}\right)$ we ask that

$$
v_{i}+\lambda \mathfrak{F}\left(v_{i}\right) \ni w_{i} \quad i=1,2 \quad \Rightarrow \quad\left\|v_{1}-v_{2}\right\| \leq\left\|w_{1}-w_{2}\right\|, \quad 0<\lambda \leq \tau,
$$

whereas for the explicit scheme $\left(\mathrm{ES}_{1}\right)$ we impose

$$
v_{i}=w_{i}-\lambda \mathfrak{F}\left(w_{i}\right) \quad i=1,2 \quad \Rightarrow \quad\left\|v_{1}-v_{2}\right\| \leq\left\|w_{1}-w_{2}\right\|, \quad 0<\lambda \leq \tau . \quad\left(\mathrm{ES}_{3}\right)
$$

It is well known that assumptions $\left(\mathrm{IS}_{2,3}\right)$ characterize the class of $m$-accretive operators in $\mathscr{B}$ and their validity extend to every $\lambda>0$; it turns out (see $\S 3$ ) that $\left(\mathrm{ES}_{3}\right)$ implies $\left(\mathrm{IS}_{3}\right)$ and that $\mathfrak{F}$ is Lipschitz continuous with constant $L_{\mathfrak{F}} \leq 2 \tau^{-1}$. Consequently, in the explicit case, we are in fact imposing a constraint $\tau \leq 2 L_{\mathfrak{F}}^{-1}$ on the maximum of the time steps, which may be viewed as an abstract $C F L$ condition: we will call an operator $\mathfrak{F}$ satisfying $\left(\mathrm{ES}_{3}\right)$ explicitly $\tau$-contractive. In any case, $\left(\mathrm{IS}_{2,3}\right)$ (and even more $\left(\mathrm{ES}_{2,3}\right)$ ) assures the existence and uniqueness of a mild/integral solution of $(\mathrm{CP})$, which yields a well posed problem $[15,4,2,30]$.

Estimates for the error $u-U$ of order $O(\sqrt{\tau})$ for $\left(\mathrm{IS}_{1}\right)$ were derived by CRAndall and Liggett [15] for uniform time-steps, and then extended to nonuniform partitions in [20]. CRANDALL and Evans [12] showed that the core of the theory lies on some estimates concerning the degree of approximation by solutions of difference schemes to the exact solution of a boundary value problem involving the differential operator $\partial / \partial s+\partial / \partial t$. All these error estimates are a priori, i.e. the rate depends on the data $f$ and $u_{0}$, but not explicitly on $U_{n}$. 
Since they are expressed in terms of $\tau$ and not $\tau_{n}$, they cannot be used to select the time-step $\tau_{n}$ according to the local behavior of $\left(\mathrm{IS}_{1}\right)$.

In this paper we adopt the opposite point of view with respect to (w.r.t.) [12]: we regard the solutions of an approximation scheme related to (CP) as continuous solutions of a suitably relaxed formulation of $(\mathrm{CP})$, which we study in an abstract form, in order to derive the main estimates in a unified way. If the method of [12] resembles the KRUŽKOV's technique of doubling the variables [22], our key idea is to double the unknowns: for us, a relaxed solution of (CP) is a couple of functions satisfying a suitable evolution dissipation inequality related to $(\mathrm{CP})$ up to an error which we call discrepancy. In this vein, a (strong or integral) solution $u$ of $(\mathrm{CP})$ is associated to a (strong or weak, respectively) relaxed solution with discrepancy 0 .

The other main feature of our construction goes back to the notion of integral solution due to BENILAN [4]: discrete solutions are then characterized by a system of evolution inequalities involving the distance from an arbitrary "test" element $V$ of $\mathscr{B}$. In the Implicit case $\left(\mathrm{IS}_{1}\right)$ we will consider the distance between the piecewise linear interpolant of the discrete values $\left\{U_{n}\right\}_{n=0}^{N}$ from $V$ as in [27]; in the Explicit case $\left(\mathrm{ES}_{1}\right)$ we will "invert" this point of view, and we will consider the piecewise linear interpolation of the values of the "discrete distances" $\left\{\| U_{n}-\right.$ $V \|\}_{n=0}^{N}$. This idea has then also been applied in the more general context of metric spaces [1, Chap. 5],[29], where vector linear interpolation is not allowed.

This new viewpoint leads to a unified treatment of existence, uniqueness, and error analysis issues via a comparison principle between strong and weak relaxed solutions (see $\S 6$ ). As a by-product we obtain novel error estimates, which address the following fundamental numerical issues:

(a) A posteriori estimates for $\|u-U\|$ : computable quantities which depend solely on time-steps $\left\{\tau_{n}\right\}_{n=1}^{N}$, discrete solution $\left\{U_{n}\right\}_{n=1}^{N}$, and data $f, u_{0}, \mathfrak{F}$;

(b) Optimal rates of convergence: a posteriori bounds converging to zero as $\tau \downarrow 0$ with an optimal rate w.r.t. the regularity of the data;

(c) Minimal regularity: for $\left(\mathrm{IS}_{1}\right) \mathfrak{F}$ is solely assumed to be an accretive operator in $\mathscr{B}$, i.e. to satisfy $\left(\mathrm{IS}_{2}\right)$ and $\left(\mathrm{IS}_{3}\right)$ (no other regularity assumptions, such as Lipschitz continuity, are made on $\mathfrak{F}$ nor on the dimension of $\mathscr{B})$; for $\left(\mathrm{ES}_{1}\right)$ $\mathfrak{F}$ is just supposed to satisfy $\left(\mathrm{ES}_{2}\right)$ and $\left(\mathrm{ES}_{3}\right)$;

(d) Uniform stability and explicit constants: the stability and error constants are uniform with respect to possible space discretizations and they are explicitly determined without need of solving any auxiliary, or dual, problem;

(e) Variable time-steps: no a priori constraints between consecutive time-steps, which could just be taylored to the a posteriori error estimators alone.

We refer to (a) and (b) collectively as optimal a posteriori error estimates.

We now state our primary results. Let $\mathcal{P}, \tau_{n}, \tau$ be defined as in (1.1), (1.2), and let

$U(t)$ be the piecewise linear interpolant of $\left\{U_{n}\right\}_{n=0}^{N}$ on the grid $\mathcal{P}$,

$\bar{U}(t), \bar{F}(t)$ be the piecewise constant functions

which take the value $U_{n}, F_{n}$ in the interval $\left(t_{n-1}, t_{n}\right]$. 
If $g \in B V(0, T ; \mathscr{B})$, then $\operatorname{Var} g$ stands for the total variation of $g$ on $[0, T]$ (see the paragraph 4.3(b) below and [7, Appendix]).

Theorem 1 (Error Estimates) Let $U_{0} \in D(\mathfrak{F})$, let $\left\{U_{n}\right\}_{n=0}^{N}$ be the solution of the implicit $\left(\mathrm{IS}_{1,2,3}\right)$ or of the explicit $\left(\mathrm{ES}_{1,2,3}\right)$ Euler scheme and let $U, \bar{F}$ be defined as in (1.4), (1.5). If $u$ is the unique mild/integral solution of $(\mathrm{CP})$, then we have the following a posteriori error estimate

$$
\begin{aligned}
\max _{0 \leq t \leq T} \|(u & -U)(t)\|\leq\| u_{0}-U_{0}\|+\| f-\bar{F} \|_{L^{1}(0, T ; \mathscr{B})} \\
& +2\left(\left\|F_{1}-\mathfrak{F}\left(U_{0}\right)\right\|+\operatorname{Var}(\bar{F})\right)^{1 / 2}\left(\sum_{n=1}^{N} \tau_{n}\left\|U_{n}-U_{n-1}\right\|\right)^{1 / 2} .
\end{aligned}
$$

Moreover, the a posteriori error estimator is bounded a priori as follows:

$$
\begin{aligned}
\sum_{n=1}^{N} \tau_{n}\left\|U_{n}-U_{n-1}\right\| & \leq T\left(\left\|F_{1}-\mathfrak{F}\left(U_{0}\right)\right\|+\operatorname{Var}(\bar{F})\right) \tau \\
& \leq T\left(\left\|f(0)-\mathfrak{F}\left(U_{0}\right)\right\|+\operatorname{Var}(f)\right) \tau,
\end{aligned}
$$

where, only for (1.8), we have chosen $F_{n}:=f\left(t_{n-1}\right)$.

As a particular case of Theorem 1, we find the a priori error estimate in the case $u_{0}=U_{0} \in D(\mathfrak{F}), f=F=0$

$$
\max _{0 \leq t \leq T}\|(u-U)(t)\| \leq 2\left\|\mathfrak{F}\left(u_{0}\right)\right\| \sqrt{T \tau} .
$$

For $\left(\mathrm{IS}_{1,2,3}\right)$, this coincides exactly with the estimate of [15, eq. (1.10)] in the case of a uniform mesh; this is also the asymptotic behavior proved by KUZNETSOV [23] for scalar conservation laws. These results extend to accretive operators in Banach spaces the optimal a posteriori error estimates derived by Nоснетто, SAVARÉ and Verdi [26, 27] for subgradient and angle-bounded operators in Hilbert spaces; the rate of convergence proved in $[26,27]$ is $O(\tau)$ provided $u_{0} \in$ $D(\mathfrak{F})$. Since such an order cannot be better than $O(\sqrt{\tau})$ for general monotone operators in Hilbert spaces [28], our present results are optimal. We refer to [25] for simpler results, and to $[1,29]$ for extensions to the Wasserstein metric.

This paper is organized as follows. In $\S 2$ we present the basic ideas leading to Theorem 1 for ODE in $\mathbb{R}^{d}$; this simplified derivation is later regarded as a reference for more technical developments. In $\S 3$ we exhibit applications of Theorem 1 to PDE such as scalar viscous conservation laws, degenerate parabolic PDEs (with or without hysteresis), and Hamilton-Jacobi equations. We recall some instrumental results in functional analysis in $\S 4$; in particular we will discuss some properties related to $\left(\mathrm{ES}_{2,3}\right)$. We motivate the notion of relaxed solution in $\S 5$ via $\left(\mathrm{IS}_{1}\right),\left(\mathrm{ES}_{1}\right)$, and the Yosida regularization of $(\mathrm{CP})$, and we prove a comparison principle for relaxed solutions in $\S 6$. In $\S 7$ we apply this comparison principle and further stability estimates to derive (a more general version of) Theorem 1 and an estimate for the Yosida regularization of (CP). They slightly extend and unify the classical approaches to existence and uniqueness of mild/integral solutions of $(\mathrm{CP})$ for $m$-accretive operators developed by Crandall, Liggett, Evans, and Bénilan. 


\section{Basic Ideas: Proof of Theorem 1 for ODE}

We consider the simplified finite dimensional setting $\mathscr{B}:=\mathbb{R}^{d}$ endowed with a differentiable (but not necessarily euclidean) norm $\|\cdot\|$. We let $j: \mathbb{R}^{d} \rightarrow \mathbb{R}^{d}$ be the differential of the norm (we formally define $j(0):=0$ ), which satisfies $\|v\|=\langle v, j(v)\rangle$ for all $v \in \mathbb{R}^{d}$, and $\mathfrak{F}: \mathbb{R}^{d} \rightarrow \mathbb{R}^{d}$ be a Lipschitz and accretive vector field, thereby satisfying

$$
\langle\mathfrak{F}(u)-\mathfrak{F}(v), j(u-v)\rangle \geq 0 \quad \forall u, v \in \mathbb{R}^{d} .
$$

Supposing for simplicity $f, F \equiv 0$, we are thus approximating the system of nonlinear ODE's

$$
u^{\prime}(t)+\mathfrak{F}(u(t))=0, \quad u(0)=u_{0}
$$

by the implicit scheme

$$
\frac{U_{n}-U_{n-1}}{\tau_{n}}+\mathfrak{F}\left(U_{n}\right)=0,
$$

which can also be rewritten as

$$
U^{\prime}(t)+\mathfrak{F}(\bar{U}(t))=0 \quad \text { a.e. in }(0, T),
$$

where $(U, \bar{U})$ is the couple of discrete solutions defined by $(1.4),(1.5)$.

Failure of the Trivial Strategy. Let us first remark that the usual strategy to derive stability estimate, i.e. "take the difference of the equations $(2.2)$ and (2.4) and multiply it by $j(u(t)-U(t))$ " does not work here since we need to multiply by $j(u(t)-\bar{U}(t))$ in order to take advantage of $(2.1)$, but with this choice the term $\left\langle u^{\prime}-U^{\prime}, j(u-\bar{U})\right\rangle$ is no longer the derivative of the norm $\|u-U\|$. If one tries to control this difference by standard perturbation arguments, the difficulty of dealing with two nonlinearities ( $\mathfrak{F}$ and $j$ ) comes up, and this does not allow for estimates independent of the dimension $d$ and of the Lipschitz constant of $\mathfrak{F}$.

Therefore, we are forced to deal with the continuous and the discrete equations separately. We describe now the crucial steps of our argument.

I. Evolution Equation for the Continuous Solution. Since $j$ is the differential of the norm, (2.2) is in fact equivalent of the system of evolution equations

$$
\frac{d}{d t}\|u(t)-v\|=-\langle\mathfrak{F}(u(t)), j(u(t)-v)\rangle \quad \forall v \in \mathbb{R}^{d},
$$

which we will try to reproduce in the discrete setting, starting from (2.4).

II. Evolution Inequalities for the Discrete Solutions. The map $s \mapsto$ $\|U(s)-V\|$ is convex in each interval $\left(t_{n-1}, t_{n}\right]$ and therefore its derivative is not decreasing and it is bounded by its value at $s=t_{n}$; in particular

$$
\begin{aligned}
\frac{d}{d s}\|U(s)-V\| & =\left\langle U^{\prime}(s), j(U(s)-V)\right\rangle \\
& \leq\left\langle U^{\prime}(s), j(\bar{U}(s)-V)\right\rangle \quad \forall V \in \mathbb{R}^{d}, \quad \text { a.e. in }(0, T) .
\end{aligned}
$$

Combining (2.4) with (2.6) we end up with

$$
\frac{d}{d s}\|U(s)-V\| \leq-\langle\mathfrak{F}(\bar{U}(s)), j(\bar{U}(s)-V)\rangle \quad \forall V \in \mathbb{R}^{d}, \quad \text { a.e. in }(0, T) .
$$


III. Doubling Variables. Now we are ready to combine (2.5) and (2.7) with (2.1): as usual in such monotonicity argument, we would like to choose the elements $v, V$ as $v:=U$ and $V:=u$ and to sum up the equations. The main point here is that it is impossible to write (2.5) and (2.7) at the same time " $t=s "$, since we derived the equations with respect to time-independent test "elements" $v, V$. Therefore we keep two distinct "time" variables $t, s$ and we choose $v:=\bar{U}(s), V:=u(t)$ obtaining, by $(2.1)$

$$
\frac{\partial}{\partial s}\|U(s)-u(t)\|+\frac{\partial}{\partial t}\|\bar{U}(s)-u(t)\| \leq 0
$$

IV. Penalization. Now we introduce a parameter $\varepsilon \in(0, T)$, and we integrate this inequality in the two-dimensional strip

$$
Q_{0, T}^{\varepsilon}:=\left\{(s, t) \in \mathbb{R}^{2}: 0 \leq t \leq T, \quad t-\varepsilon \leq s \leq t\right\} .
$$

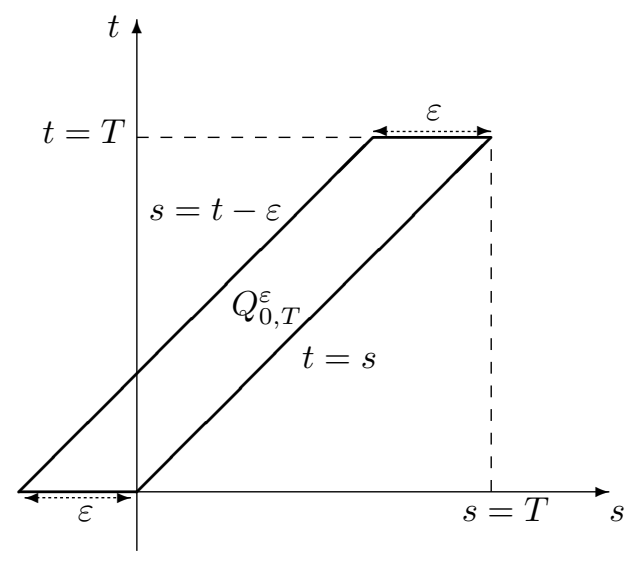

Figure 1: Strip $Q_{0, T}^{\varepsilon}$ corresponding to penalization about the diagonal $\{t=s\}$.

In order to deal with negative values of $s$, we extend $U, \bar{U}$ in $(-\varepsilon, 0)$ by

$$
\bar{U}(s):=U_{0}, \quad U(s):=U_{0}-s \mathfrak{F}\left(U_{0}\right)
$$

so that (2.4) still holds. Applying the Gauss-Green formula in $Q_{0, T}^{\varepsilon}$ gives

$$
\begin{aligned}
\int_{T-\varepsilon}^{T} \| \bar{U}(s) & -u(T)\|d s \leq \varepsilon\| u_{0}-U_{0} \| \\
& +\int_{0}^{T}(\|\bar{U}(t)-u(t)\|-\|U(t)-u(t)\|) d t \\
& +\int_{0}^{T}(\|U(t-\varepsilon)-u(t)\|-\|\bar{U}(t-\varepsilon)-u(t)\|) d t
\end{aligned}
$$

V. Stability. We take the difference between two consecutive equations (2.3), and multiply by $j\left(U_{n}-U_{n-1}\right)$, to arrive at

$$
\begin{aligned}
\frac{1}{\tau_{n}}\left\langle U_{n}-U_{n-1}, j\left(U_{n}-U_{n-1}\right)\right\rangle & +\left\langle\mathfrak{F}\left(U_{n}\right)-\mathfrak{F}\left(U_{n-1}\right), j\left(U_{n}-U_{n-1}\right)\right\rangle \\
& =\frac{1}{\tau_{n-1}}\left\langle U_{n-1}-U_{n-2}, j\left(U_{n}-U_{n-1}\right)\right\rangle .
\end{aligned}
$$


Making use of (2.1), together with property $\langle v, j(w)\rangle \leq\|v\|$ for all $v \in \mathbb{R}^{d}$ with equality for $w=v$, we get

$$
\frac{1}{\tau_{n}}\left\|U_{n}-U_{n-1}\right\| \leq \frac{1}{\tau_{n-1}}\left\|U_{n-1}-U_{n-2}\right\|, \quad \forall 1 \leq n \leq N .
$$

If we now choose $U_{-1}=U_{0}-\tau_{0} \mathfrak{F}\left(U_{0}\right)$ with $\tau_{0} \leq \varepsilon$, which is consistent with (2.10), recursion yields the following stability estimate for the discrete derivative:

$$
\sup _{t \in(0, T)}\left\|U^{\prime}(t)\right\|=\sup _{1 \leq n \leq N} \frac{1}{\tau_{n}}\left\|U_{n}-U_{n-1}\right\| \leq\left\|\mathfrak{F}\left(U_{0}\right)\right\| .
$$

VI. A Posteriori Error Estimate. Since

$$
\begin{aligned}
\|\bar{U}(t)-u(t)\|-\|U(t)-u(t)\| & \leq\|\bar{U}(t)-U(t)\|, \\
\|U(t-\varepsilon)-u(t)\|-\|\bar{U}(t-\varepsilon)-u(t)\| & \leq\|\bar{U}(t-\varepsilon)-U(t-\varepsilon)\|,
\end{aligned}
$$

and $\int_{-\varepsilon}^{0}\|\bar{U}(t)-U(t)\| d t=\frac{\varepsilon}{2}\left\|\mathfrak{F}\left(U_{0}\right)\right\|$, a simple manipulation of (2.11) implies

$$
\begin{aligned}
\frac{1}{\varepsilon} \int_{T-\varepsilon}^{T}\|U(s)-u(T)\| d s & \leq \frac{1}{\varepsilon} \int_{T-\varepsilon}^{T}(\|\bar{U}(s)-u(T)\|+\|\bar{U}(s)-U(s)\|) d s \\
& \leq\left\|u_{0}-U_{0}\right\|+\frac{\varepsilon}{2}\left\|\mathfrak{F}\left(U_{0}\right)\right\|+\frac{2}{\varepsilon} \int_{0}^{T}\|\bar{U}(t)-U(t)\| d t .
\end{aligned}
$$

Since (1.4) and (1.5) imply

$$
\int_{0}^{T}\|U(t)-\bar{U}(t)\| d t=\frac{1}{2} \sum_{n=1}^{N} \tau_{n}\left\|U_{n}-U_{n-1}\right\|,
$$

and (2.12) leads to

$$
\frac{1}{\varepsilon} \int_{T-\varepsilon}^{T}\|U(s)-U(T)\| d s \leq \frac{\varepsilon}{2}\left\|\mathfrak{F}\left(U_{0}\right)\right\|,
$$

we readily infer that

$$
\|u(T)-U(T)\| \leq\left\|u_{0}-U_{0}\right\|+\varepsilon\left\|\mathfrak{F}\left(U_{0}\right)\right\|+\frac{1}{\varepsilon} \sum_{n=1}^{N} \tau_{n}\left\|U_{n}-U_{n-1}\right\| .
$$

Upon optimizing $\varepsilon$, we conclude the desired a posteriori error bound

$$
\|u(T)-U(T)\| \leq\left\|u_{0}-U_{0}\right\|+2\left\|\mathfrak{F}\left(U_{0}\right)\right\|^{1 / 2}\left(\sum_{n=1}^{N} \tau_{n}\left\|U_{n}-U_{n-1}\right\|\right)^{1 / 2} .
$$

VII. A Priori Error Estimate. To show that the above error bound exhibits the correct asymptotic order of convergence, we resort to (2.12) to deduce

$$
\sum_{n=1}^{N} \tau_{n}\left\|U_{n}-U_{n-1}\right\| \leq \tau T\left\|\mathfrak{F}\left(U_{0}\right)\right\| .
$$

This yields the following celebrated a priori estimate of CRANDALL and LiGGETT [15], originally derived for constant time steps:

$$
\|u(T)-U(T)\| \leq\left\|u_{0}-U_{0}\right\|+2\left\|\mathfrak{F}\left(U_{0}\right)\right\| \sqrt{\tau T} .
$$




\section{Applications of Theorem 1 to PDE}

In this section we present several concrete examples. We provide some basic background and references where the assumptions $\left(\mathrm{IS}_{2}\right)$ and $\left(\mathrm{IS}_{3}\right)$ are shown.

In some case it is easier to define $\mathfrak{F}$ by a closure procedure, starting from a smaller selection $\mathfrak{F}_{0}$ defined in a subset $D\left(\mathfrak{F}_{0}\right)$ of a new Banach space $\mathscr{B}_{0} \subset \mathscr{B}$ dense in $\mathscr{B}$. More precisely, if $\mathfrak{F}_{0}: D\left(\mathfrak{F}_{0}\right) \subset \mathscr{B}_{0} \rightarrow 2^{\mathscr{B}_{0}}$ is a multivalued operator satisfying

$$
\begin{gathered}
\forall w \in \mathscr{B}_{0}, \lambda>0 \quad \exists v \in D\left(\mathfrak{F}_{0}\right): v+\lambda \mathfrak{F}_{0}(v) \ni w, \\
v_{i} \in D\left(\mathfrak{F}_{0}\right), v_{i}+\lambda \mathfrak{F}_{0}\left(v_{i}\right) \ni w_{i} \in \mathscr{B}_{0} \quad \Rightarrow \quad\left\|v_{1}-v_{2}\right\| \leq\left\|w_{1}-w_{2}\right\|,
\end{gathered}
$$

then it is not difficult to see that the strong closure of the graph of $\mathfrak{F}_{0}$ in $\mathscr{B} \times \mathscr{B}$ produces an $m$-accretive operator $\mathfrak{F}$, which is therefore defined as

$$
w \in \mathfrak{F}(v) \Leftrightarrow \exists w_{n} \in \mathfrak{F}_{0}\left(v_{n}\right):\left(v_{n}, w_{n}\right) \rightarrow(v, w) \text { strongly in } \mathscr{B} \times \mathscr{B} .
$$

\subsection{Scalar Conservation Laws}

Consider the Cauchy problem for the following viscous conservation law in $\mathbb{R}^{d}$

$$
\partial_{t} u+\operatorname{div} \phi(u)-\epsilon \Delta u=f, \quad u(0, \cdot)=u_{0},
$$

where $\epsilon>0, u_{0} \in L^{1}\left(\mathbb{R}^{d}\right) \cap L^{\infty}\left(\mathbb{R}^{d}\right)$, and the nonlinear function $\phi$ satisfies

$$
\phi \in C^{1}\left(\mathbb{R}, \mathbb{R}^{d}\right), \quad \phi(0)=0 .
$$

Consider the Banach spaces $\mathscr{B}:=L^{1}\left(\mathbb{R}^{d}\right), \mathscr{B}_{0}:=L^{1}\left(\mathbb{R}^{d}\right) \cap L^{\infty}\left(\mathbb{R}^{d}\right)$ and the operator $\mathfrak{F}_{0}(v):=\operatorname{div} \phi(v)-\epsilon \Delta v$ with domain

$$
D\left(\mathfrak{F}_{0}\right):=\left\{v \in \mathscr{B}_{0} \cap H^{2}\left(\mathbb{R}^{d}\right): \mathfrak{F}_{0}(v) \in \mathscr{B}_{0}\right\} .
$$

Then $\mathfrak{F}_{0}$ satisfies (3.1a) and (3.1b) [11, Prop. 2.2, Lemma 2.4, Cor. 2.2] and $u=u(x, t)$ is a bounded variational solution of (3.3) if and only if $t \mapsto u(\cdot, t)$ solve the abstract $(\mathrm{CP})$ for $\mathfrak{F}$ given as in (3.2).

The limit case $\epsilon=0$ is more delicate and was first studied by KRUŽKOV [22], and later by CRANDALL [11] within the context of accretive operators in $L^{1}$. The operator $\mathfrak{F}_{0}$ is now defined [11, Def. 1.1, Cor. 2.2] on $v \in \mathscr{B}_{0}=L^{1}\left(\mathbb{R}^{d}\right) \cap L^{\infty}\left(\mathbb{R}^{d}\right)$ as the set of $w \in \mathscr{B}_{0}$ such that as

$$
\int_{\mathbb{R}^{d}} \operatorname{sign}_{0}(v(x)-k)((\phi(v(x))-\phi(k)) \nabla \varphi(x)+w(x) \varphi(x)) d x \geq 0
$$

for all $\varphi \in C_{0}^{\infty}\left(\mathbb{R}^{d}\right), \varphi \geq 0$, and $k \in \mathbb{R}$; here

$$
\operatorname{sign}_{0}(0)=0, \quad \operatorname{sign}_{0}(x)=\frac{x}{|x|} \text { if } x \neq 0 .
$$

As before, $u(x, t)$ is a bounded entropy solution of $(3.3)$ for $\varepsilon=0$ if $t \mapsto u(\cdot, t)$ is an integral solution of $(\mathrm{CP})$.

In both cases, $\epsilon>0$ and $\epsilon=0$, the operator $\mathfrak{F}$ is $m$-accretive in $\mathscr{B}$. Our error estimates of Theorem 1 for the scheme

$$
\frac{U_{n}-U_{n-1}}{\tau_{n}}+\operatorname{div} \phi\left(U_{n}\right)-\varepsilon \Delta U_{n}=F_{n}
$$

are thus uniform in the viscosity parameter $\epsilon$. 


\subsection{A Finite Volume Method for Conservation Laws}

Let $\mathcal{T}$ be decomposition of a bounded domain of $\mathbb{R}^{d}$ into simplices $K$ and let $d$ be the cardinal of $\mathcal{T}$. For each pair of adjacent volumes $K, L \in \mathcal{T}$ there is a discrete flux $g_{K, L} \in C^{0}\left(\mathbb{R}^{2}\right)$ satisfying the structural properties

$$
g_{K, L}(u, v)=-g_{L, K}(v, u)
$$

$g_{K, L}$ is increasing w.r.t. $u$ and decreasing w.r.t. $v$;

we set $g_{K, L}(u, v) \equiv 0$ if $K, L$ are not adjacent [19], [21]. We consider the following semidiscrete piecewise constant finite volume discretization of (3.3) with $\epsilon=0$. Let $u(t):=\left\{u_{K}(t)\right\}_{K \in \mathcal{T}}$ be the semidiscrete solution of

$$
|K| \frac{d}{d t} U_{K}(t)+\sum_{L \in \mathcal{T}}|K| L\left|g_{K, L}\left(U_{K}(t), U_{L}(t)\right)=\right| K \mid f_{K}(t),
$$

where $|K| L \mid$ stands for the measure of the common side between $K$ and $L$ $(|K| L \mid=0$ if $K, L$ are disjoint). The implicit version of (3.11) reads

$$
|K| \frac{U_{n, K}-U_{n-1, K}}{\tau_{n}}+\sum_{L \in \mathcal{T}}|K| L\left|g_{K, L}\left(U_{n, K}, U_{n, L}\right)=\right| K \mid F_{n, K} .
$$

If $u:=\left\{u_{K}\right\}_{K \in \mathcal{T}}, U_{n}:=\left\{U_{n, K}\right\}_{K \in \mathcal{T}}$, and $\mathfrak{F}(U):=\left\{\mathfrak{F}_{K}(U)\right\}_{K \in \mathcal{T}}: \mathbb{R}^{d} \rightarrow \mathbb{R}^{d}$ is defined by

$$
\mathfrak{F}_{K}(U):=\frac{1}{|K|} \sum_{L \in \mathcal{T}}|K| L \mid g_{K, L}\left(U_{K}, U_{L}\right) \quad \forall U:=\left\{U_{K}\right\}_{K \in \mathcal{T}} \in \mathbb{R}^{d},
$$

then $(3.11)$ and $(3.12)$ correspond to $(\mathrm{CP})$ and $\left(\mathrm{IS}_{1}\right)$ respectively for $\mathfrak{F}$. Observe that (3.9) yields the properties

$$
\begin{gathered}
\sum_{K \in \mathcal{T}}|K| \mathfrak{F}_{K}(U)=0 \\
\sum_{K \in \mathcal{T}}|K| U_{n, K}=\sum_{K \in \mathcal{T}}|K| U_{n-1, K}+\tau_{n} \sum_{K \in \mathcal{T}}|K| F_{n, K} .
\end{gathered}
$$

Let us show that (3.9) and (3.10) imply $\mathfrak{F}$ is $m$-accretive w.r.t. the $L^{1}$-type norm $\|v\|_{1}:=\sum_{K \in \mathcal{T}}|K|\left|U_{K}\right|$ in $\mathbb{R}^{d}$. Since $\mathfrak{F}$ is continuous, it is enough to check accretivity. We invoke an equivalent characterization of accretivity via the so called duality map $J: \mathbb{R}^{d} \rightarrow 2^{\mathbb{R}^{d}}$ (see (4.1) and (4.11) in $\S 3$ ): setting (see (3.7))

$$
J_{0}(U):=\left\{\operatorname{sign}_{0}\left(U_{K}\right)\right\}_{K \in \mathcal{T}}, \quad \text { which belongs to } J(U),
$$

the accretivity of $\mathfrak{F}$ follows provided we show that for all $U, V$

$$
\left\langle\mathfrak{F}(U)-\mathfrak{F}(V), J_{0}(U-V)\right\rangle=\sum_{K \in \mathcal{T}}\left(\mathfrak{F}_{K}(U)-\mathfrak{F}_{K}(V)\right) \operatorname{sign}_{0}\left(U_{K}-V_{K}\right) \geq 0 .
$$

By (3.9) we have

$$
\begin{aligned}
\langle\mathfrak{F}(U)-\mathfrak{F}(V), & \left.J_{0}(U-V)\right\rangle= \\
& =\sum_{K \in \mathcal{T}}\left(\sum_{L \in \mathcal{T}} g_{K, L}\left(U_{K}, U_{L}\right)-g_{K, L}\left(V_{K}, V_{L}\right)\right) \operatorname{sign}_{0}\left(U_{K}-V_{K}\right) \\
& =-\sum_{K \in \mathcal{T}}\left(\sum_{L \in \mathcal{T}} g_{L, K}\left(U_{L}, U_{K}\right)-g_{L, K}\left(V_{L}, V_{K}\right)\right) \operatorname{sign}_{0}\left(U_{K}-V_{K}\right) .
\end{aligned}
$$


Consequently, if

$$
\begin{aligned}
& A_{K, L}:=g_{K, L}\left(U_{K}, U_{L}\right)-g_{K, L}\left(V_{K}, V_{L}\right), \\
& B_{K, L}:=\operatorname{sign}_{0}\left(U_{K}-V_{K}\right)-\operatorname{sign}_{0}\left(U_{L}-V_{L}\right),
\end{aligned}
$$

we then infer (3.15) because

$$
\left\langle\mathfrak{F}(U)-\mathfrak{F}(V), J_{0}(U-V)\right\rangle=\frac{1}{2} \sum_{K, L \in \mathcal{T}} A_{K, L} B_{K, L} \geq 0 .
$$

If $W_{K}:=U_{K}-V_{K}$, the last inequality results from (3.10) and the properties:

$$
\begin{aligned}
W_{K}, W_{L}>0 & \Rightarrow \quad B_{K, L}=0 \\
W_{K}, W_{L}<0 & \Rightarrow \quad B_{K, L}=0 \\
W_{K},-W_{L} \leq 0 & \Rightarrow \quad B_{K, L} \leq 0, A_{K, L} \leq 0 \\
W_{K},-W_{L} \geq 0 & \Rightarrow \quad B_{K, L} \geq 0, A_{K, L} \geq 0
\end{aligned}
$$

Since $\mathfrak{F}$ is $m$-accretive in $\mathbb{R}^{d}$ w.r.t. the norm $\|\cdot\|_{1}$, our error estimates of Theorem 11 are valid for this fully discrete finite volume method.

Let us now switch to the explicit scheme

$$
|K| \frac{U_{n, K}-U_{n-1, K}}{\tau_{n}}+\sum_{L \in \mathcal{T}}|K| L \mid g_{K, L}\left(U_{n-1, K}, U_{n-1, L}\right)=0 .
$$

In order to apply Theorem 1 , we have to check if $\mathfrak{F}$ is explicitly $\tau$ contractive for some $\tau>0$. Besides (3.9) and (3.10), we add now that the flux functions $g_{K, L}$ are Lipschitz continuous, i.e.

$$
\left|g_{K, L}\left(u_{1}, v\right)-g_{K_{L}}\left(u_{2}, v\right)\right| \leq \lambda_{K, L}\left|u_{1}-u_{2}\right| \quad \forall u_{1}, u_{2}, v \in \mathbb{R} .
$$

If we set

$$
\lambda_{K}:=\frac{1}{|K|} \sum_{L \in \mathcal{T}}|K| L \mid \lambda_{K, L}, \quad \lambda:=\sup _{K \in \mathcal{T}} \lambda_{K},
$$

then it is possible to prove that

$$
\text { if } \tau \lambda \leq 1 \text { then } \mathfrak{F} \text { is explicitly } \tau \text { contractive. }
$$

\subsection{Degenerate Parabolic Equations}

Let $\Omega$ be a smooth bounded domain of $\mathbb{R}^{d}, f \in B V(0, T ; \mathscr{B})$ with $\mathscr{B}:=L^{1}(\Omega)$. Consider the initial-boundary problem for the following degenerate parabolic equation in $\Omega$

$$
\partial_{t} u-\Delta \beta(u)=f, \quad u(0, \cdot)=u_{0},
$$

with $\beta$ a maximal monotone graph in $\mathbb{R}^{2}$ such that $0 \in \beta(0)$. The operator $\mathfrak{F}(v):=-\Delta \beta(v)$, defined in $D(\mathfrak{F}):=\left\{v \in L^{1}(\Omega): \beta(v) \in W_{0}^{1,1}(\Omega), \Delta \beta(v) \in\right.$ $\left.L^{1}(\Omega)\right\}$, was shown to be $m$-accretive by BrEzIS and STRAuss [9]; see also [30]. This setting models a number of important physical processes. Within the class $\beta \in W^{1, \infty}(\mathbb{R}, \mathbb{R})$, we have the Stefan problem for which $\beta(s)=s^{-}+(1-s)^{+}$. If, instead, $\beta^{-1}(s)=1-(s-1)^{-}$we have an elliptic-parabolic equation which describes filtration with partial saturation. 
Our error estimates of Theorem 1 apply to the scheme

$$
\frac{U_{n}-U_{n-1}}{\tau_{n}}-\Delta \beta\left(U_{n}\right)=F_{n}
$$

irrespective of the regularity of the maximal monotone graphs $\beta$ or of the solutions.

Equations (3.3) and (3.21) may be combined together in $\mathbb{R}^{d}$

$$
\partial_{t} u+\operatorname{div} \phi(u)-\Delta \beta(u)=f, \quad u(0, \cdot)=u_{0} .
$$

It is shown by COCKBURN and GRIPENBERG [10] that under suitable assumptions on $\phi$ and $\beta$ the ensuing operator $\mathfrak{F}(v):=\operatorname{div} \phi(u)-\Delta \beta(u)$ is $m$-accretive in $\mathscr{B}$. Therefore our error estimates are also valid in this case for the related scheme.

\subsection{Parabolic Equations with Hysteresis}

We describe briefly a model due to Visintin [31]. Let $\gamma_{-}, \gamma_{+}$be two maximal monotone (possibly multivalued) graphs in $\mathbb{R}^{2}$ such that

$$
\inf \gamma_{-}(v) \leq \sup \gamma_{+}(v) \quad \forall v \in \mathbb{R}
$$

and let $\varphi(v, w)$ be the hysteresis loop in $\mathbb{R}^{2}$

$$
\varphi(v, w):= \begin{cases}\{+\infty\} & \text { if } w<\inf \gamma_{+}(v), \\ {[0, \infty]} & \text { if } w \in \gamma_{-}(v) \backslash \gamma_{+}(v), \\ \{0\} & \text { if } \sup \gamma_{-}(v)<w<\gamma_{+}(v), \\ {[-\infty, 0]} & \text { if } w \in \gamma_{-}(v) \backslash \gamma_{+}(v), \\ \{-\infty\} & \text { if } w>\sup \gamma_{-}(v), \\ {[-\infty,+\infty]} & \text { if } w \in \gamma_{-}(v) \cap \gamma_{+}(v)\end{cases}
$$

Let $\Omega$ and $f$ be as in $\S 3.3$. We consider the parabolic system in $\Omega \times(0, T)$ :

$$
\partial_{t} u+\xi-\Delta u=f, \quad \partial_{t} w-\xi=0, \quad \xi \in \varphi(u, w),
$$

with Dirichlet boundary condition for $u$ and initial condition $u_{0}, w_{0}$. Equivalently, if $V:=(u, w)$ and $F:=(f, 0)$, we can write (3.25) in vector form

$$
\partial_{t} V+\mathcal{A}(V)+L V \ni F \text {, }
$$

where

$$
\left\{\begin{array}{l}
D(\mathcal{A}):=\left\{V=(u, w) \in \mathbb{R}^{2}: \inf \gamma_{-}(u) \leq w \leq \sup \gamma_{+}(u)\right\}(=D(\varphi)), \\
\mathcal{A}(V):=\{(\xi,-\xi): \xi \in \varphi(V) \cap \mathbb{R}\} \quad \forall V \in D(\mathcal{A})
\end{array}\right.
$$

and

$$
L V:=(-\Delta u, 0), \quad D(L):=\left\{(u, w) \in L^{1}\left(\Omega ; \mathbb{R}^{2}\right): u \in W_{0}^{1,1}(\Omega), \Delta u \in L^{1}(\Omega)\right\} .
$$

If $D(\mathcal{A}) \neq \emptyset$, then $\mathcal{A}$ is $m$-accretive in $\mathbb{R}^{2}$ [31]; this follows from an argument similar to that in (3.16). Moreover, if $\gamma_{-}, \gamma_{+}$do not grow faster than linearly at $\pm \infty$, namely $|z| \leq C_{1}|v|+C_{2}$ for all $v \in \mathbb{R}$ and $z \in \gamma_{-}(v), \gamma_{+}(v)$ with constants $C_{1}, C_{2}>0$, then the nonlinear (multivalued) operator $\mathfrak{F}:=\mathcal{A}+L$ is $m$-accretive in $L^{1}\left(\Omega ; \mathbb{R}^{2}\right)$. Our error estimates of Theorem 1 are valid for (3.26) and are the first ones for this problem. 


\subsection{Hamilton-Jacobi Equations}

Let $H \in C(\mathbb{R}, \mathbb{R})$ be a Hamiltonian and let $\mathscr{B}=\mathrm{BUC}\left(\mathbb{R}^{d}\right)$ be the space of bounded uniformly continuous functions over $\mathbb{R}^{d}$ with the "sup" norm. We consider the Cauchy problem

$$
\partial_{t} u+H(\nabla u)=f, \quad u(\cdot, 0)=u_{0},
$$

with $u_{0} \in \mathscr{B}$. Viscosity solutions of (3.27) have been constructed by CRANDALL, Evans and Lions [13], [16]; see also [14]. If we define the domain $D(\mathfrak{F})$ of $\mathfrak{F}$ as the set of all viscosity solutions $u \in \mathscr{B}$ of $H(\nabla u)=f$ for some $f \in \mathscr{B}$, and $\mathfrak{F}(u) \in \mathscr{B}$ the set of all such $f$, then $\mathfrak{F}$ is $m$-accretive in $\mathscr{B}[13]$.

Therefore, our error estimates of Theorem 1 apply to the scheme

$$
\frac{U_{n}-U_{n-1}}{\tau_{n}}+H\left(\nabla U_{n}\right)=F_{n} .
$$

A general way to approximate (3.27) [3] by a so called "monotone scheme" is to introduce a family of maps $S(h): B\left(\mathbb{R}^{d}\right) \rightarrow B\left(\mathbb{R}^{d}\right), h>0$, (here $B\left(\mathbb{R}^{d}\right)$ denotes the space of bounded real functions defined on $\mathbb{R}^{d}$ ) which satisfies the properties

$$
\begin{aligned}
& u \geq v \Rightarrow S(h) u \geq S(h) v \quad \forall u, v \in B\left(\mathbb{R}^{d}\right), \\
& S(h)(u+k)=S(h) u+k \quad \forall u \in B\left(\mathbb{R}^{d}\right), k \in \mathbb{R}, \\
& \frac{\phi-S(h) \phi}{h} \rightarrow H(\nabla \phi) \quad \text { as } h \downarrow 0 \quad \forall \phi \in C^{\infty}\left(\mathbb{R}^{d}\right) \cap B\left(\mathbb{R}^{d}\right) .
\end{aligned}
$$

In this approach, the approximation scheme is given by $U_{0}:=u_{0}$ and

$$
U_{n}=\left(1-\frac{\tau_{n}}{h}\right) U_{n-1}+\frac{\tau_{n}}{h} S(h) U_{n-1}+\tau_{n} F_{n},
$$

which correspond to $\left(\mathrm{ES}_{1}\right)$ for the operator

$$
\mathfrak{F}^{h}(v):=\frac{v-S(h) v}{h} .
$$

A result of Crandall and Tartar [17] (see also paragraph 4.2(b)) shows that $\mathfrak{F}^{h}$ satisfies $\left(\mathrm{ES}_{3}\right)$ for $\tau=\max _{n} \tau_{n} \leq h$. Theorem 1 can thus be applied and provides a flexible choice of the mesh $\mathcal{\mathcal { P }}$, satisfying the CFL-like condition $\tau \leq h$.

\section{Differential Calculus in Banach Spaces}

In this section we collect some basic functional analytic facts of Banach spaces that will be instrumental in the subsequent discussion. We refer to [2], [18], [24], [30] for more details and proofs.

Notation 4.1 (Multivalued Maps) We will often deal with multivalued maps $\mathfrak{J}: X \rightarrow 2^{Y}, X, Y$ being given sets: we will use the symbol $\mathfrak{J}(x)$ to indicate any selection $y \in \mathfrak{J}(x)$, the same at every occurence of $\mathfrak{J}(x)$ in a given formula. 


\subsection{Accretive Operators.}

4.1(a) Duality Map. We will denote by $\mathscr{B}^{*}$ the dual space of $\mathscr{B}$, endowed with the dual norm $\|\cdot\|_{*} ;\langle\cdot, \cdot\rangle$ is the duality pairing between $\mathscr{B}$ and $\mathscr{B}^{*}$. The duality map [30, p. 91] $J: \mathscr{B} \rightarrow 2^{\mathscr{B}^{*}}$ is defined by

$$
J(v)=\left\{v^{*} \in \mathscr{B}^{*}:\left\|v^{*}\right\|_{*} \leq 1,\left\langle v, v^{*}\right\rangle=\|v\|\right\} ;
$$

for every $v \in \mathscr{B}, J(v)$ is a nonempty, weakly* compact, and convex set of $\mathscr{B}^{*}$, with $\|J(v)\|_{*}=1$ if $v \neq 0$. We present now three relevant examples.

1. If $\mathscr{B}$ is a Hilbert space, then we can identify $\mathscr{B}^{*}$ with $\mathscr{B}$ and in this case $J(v)=\frac{v}{\|v\|}$ for every $v \neq 0$.

2. If $\mathscr{B}=L^{1}(\Omega), \Omega$ being an open subset of $\mathbb{R}^{d}$, then $\mathscr{B}^{*}=L^{\infty}(\Omega)$ and $J(v)=\operatorname{sign}(v)$.

3. If $\mathscr{B}=C_{0}^{0}(\Omega)$ is the completion in the maximum norm of the space of continuous functions with compact support, then $\mathscr{B}^{*}=M(\Omega)$ is the space of finite (signed) measures $\mu$ with Jordan decomposition $\mu=\mu^{+}-\mu^{-}$and, for $v \not \equiv 0$,

$$
\mu \in J(v) \Leftrightarrow\left\{\begin{array}{l}
\mu^{+}(\Omega)+\mu^{-}(\Omega)=1 \\
\operatorname{supp} \mu^{+} \subset\left\{x \in \Omega: v(x)=\|v\|_{C_{0}^{0}(\Omega)}\right\}, \\
\operatorname{supp} \mu^{-} \subset\left\{x \in \Omega: v(x)=-\|v\|_{C_{0}^{0}(\Omega)}\right\} .
\end{array}\right.
$$

We thus see that $J(v)$ is composed of measures with unit mass and support in the set of extremal points of $|v|$.

Examples 2 and 3 show that the duality map $J$ is in general multivalued.

4.1(b) Directional Derivatives of the Norm, Pseudo-Scalar Product. The duality map is strictly related to the differentiability properties of the norm of $\mathscr{B}$. First of all, we note that the map $\lambda \in \mathbb{R} \mapsto\|w+\lambda v\|$ is convex for all $v, w \in \mathscr{B}$. Then, the directional derivatives of the norm satisfy:

$$
\begin{aligned}
& {[v, w]_{+}:=\lim _{\lambda \downarrow 0} \frac{\|w+\lambda v\|-\|w\|}{\lambda}=\inf _{\lambda>0} \frac{\|w+\lambda v\|-\|w\|}{\lambda},} \\
& {[v, w]_{-}:=\lim _{\lambda \downarrow 0} \frac{\|w\|-\|w-\lambda v\|}{\lambda}=\sup _{\lambda>0} \frac{\|w\|-\|w-\lambda v\|}{\lambda} ;}
\end{aligned}
$$

$[\cdot, \cdot]_{ \pm}$are called pseudo-scalar products. We observe that

$$
[v, w]_{+}=[-v,-w]_{+}=-[-v, w]_{-}=-[v,-w]_{-},
$$

and

$$
\left|[v, w]_{ \pm}\right| \leq\|v\|, \quad[v, v]_{-}=[v, v]_{+}=\|v\| \quad \forall v, w \in \mathscr{B} .
$$

The duality map and the pseudo-scalar product are related by

$$
[v, w]_{-}=\min _{w^{*} \in J(w)}\left\langle v, w^{*}\right\rangle, \quad[v, w]_{+}=\max _{w^{*} \in J(w)}\left\langle v, w^{*}\right\rangle .
$$


In light of (4.2), (4.5) shows that $J$ is the subdifferential of the norm $\|\cdot\|$ of $\mathscr{B}$ [2, Chap. II, 2.2]. Moreover, we have the sub-superadditivity properties

$$
\begin{aligned}
{[v, z]_{-}+[w, z]_{-} } & \leq[v+w, z]_{-} \\
& \leq[v, z]_{-}+[w, z]_{+} \\
& \leq[v+w, z]_{+} \leq[v, z]_{+}+[w, z]_{+} \quad \forall v, w, z \in \mathscr{B} .
\end{aligned}
$$

If $t \in[0, T] \mapsto u(t) \in \mathscr{B}$ is an absolutely continuous and almost everywhere differentiable map, using (4.2) we see that

$$
\begin{aligned}
\frac{d}{d t}\|u(t)-v\| & =\left[u^{\prime}(t), u(t)-v\right]_{-} \\
& =\left[u^{\prime}(t), u(t)-v\right]_{+} \quad \forall v \in \mathscr{B}, \text { a.e. on }(0, T) .
\end{aligned}
$$

Since $[\cdot, \cdot]_{-}\left(\right.$resp. $\left.[\cdot, \cdot]_{+}\right)$is the supremum (resp. the infimum) of a family of maps which are contractions with respect to the first argument and continuous w.r.t. the second one, it is also 1-Lipschitz continuous w.r.t. to the first argument and and l.s.c. (resp. u.s.c.) with respect to the second one, i.e.

$$
\begin{gathered}
\left|[v, z]_{-}-[w, z]_{-}\right| \leq\|v-w\|, \quad\left|[v, z]_{+}-[w, z]_{+}\right| \leq\|v-w\|, \\
\lim _{n \uparrow+\infty} w_{n}=w \Rightarrow\left\{\begin{array}{l}
\liminf _{n \uparrow+\infty}\left[v, w_{n}\right]_{-} \geq[v, w]_{-}, \\
\limsup _{n \uparrow+\infty}\left[v, w_{n}\right]_{+} \leq[v, w]_{+} .
\end{array}\right.
\end{gathered}
$$

4.1(c) Accretive Operators. Let $\mathfrak{F}: \mathscr{B} \rightarrow 2^{\mathscr{B}}$ be a multi-valued operator with proper and nonempty domain

$$
D(\mathfrak{F}):=\{v \in \mathscr{B}: \mathfrak{F}(v) \neq \varnothing\} .
$$

With Notation 4.1 in mind, we see that $\mathfrak{F}$ is accretive, i.e. it satisfies $\left(\mathrm{IS}_{3}\right)$, if and only if

$$
\left[\mathfrak{F}\left(v_{1}\right)-\mathfrak{F}\left(v_{2}\right), v_{1}-v_{2}\right]_{+} \geq 0 \quad \forall v_{1}, v_{2} \in D(\mathfrak{F}) .
$$

This characterization cne be written in terms of the duality map $J$ via (4.5) as follows

$$
\forall w_{i} \in \mathfrak{F}\left(v_{i}\right), i=1,2, \quad \exists z^{*} \in J\left(v_{1}-v_{2}\right): \quad\left\langle w_{1}-w_{2}, z^{*}\right\rangle \geq 0 .
$$

Observe that the map

$$
\lambda \mapsto\left\|v_{1}-v_{2}+\lambda\left(\mathfrak{F}\left(v_{1}\right)-\mathfrak{F}\left(v_{2}\right)\right)\right\| \quad \text { is not decreasing in }[0,+\infty),
$$

because it is convex and has a minimum at $\lambda=0$. if

$\mathfrak{F}$ is closed if its graph is a closed subset of $\mathscr{B} \times \mathscr{B}$. An accretive $\mathfrak{F}$ is maximal

$$
[f-\mathfrak{F}(w), v-w]_{+} \geq 0 \quad \forall w \in D(\mathfrak{F}) \quad \Rightarrow \quad f \in \mathfrak{F}(v) .
$$

Finally, as mentioned in the introduction, $\mathfrak{F}$ is $m$-accretive if (4.10) holds and

$$
\forall w \in \mathscr{B}, \forall \varepsilon>0 \quad \exists v \in D(\mathfrak{F}): \quad v+\varepsilon \mathfrak{F}(v) \ni w .
$$

Every $m$-accretive operator is also maximal-accretive [30, Chap. IV, Prop. 7.2] (the converse is false in general, but it is true in the framework of Hilbert spaces) and every maximal-accretive operator is closed. 
4.1(d) Yosida Regularization. For $\tau>0$ we introduce the resolvent operator

$$
\mathfrak{J}_{\tau}:=(I+\tau \mathfrak{F})^{-1}, \quad v_{\tau}=\mathfrak{J}_{\tau}(v) \quad \Leftrightarrow \quad v_{\tau}+\tau \mathfrak{F}\left(v_{\tau}\right) \ni v
$$

which is a contraction of $\mathscr{B}$, and the Yosida regularization of $\mathfrak{F}$ defined as

$$
\mathfrak{F}_{\tau}: v \in \mathscr{B} \rightarrow \frac{v-\mathfrak{J}_{\tau}(v)}{\tau}, \quad \text { with the property } \quad \mathfrak{F}_{\tau}(v) \in \mathfrak{F}\left(\mathfrak{J}_{\tau}(v)\right) .
$$

It is well known that $\mathfrak{F}_{\tau}$ is a $2 \tau^{-1}$-Lipschitz accretive operator on $\mathscr{B}$ [30, Proposition 7.1]. Moreover, writing $v_{\tau}+\tau \mathfrak{F}\left(v_{\tau}\right) \ni v$, subtracting the trivial identity $v+\tau \mathfrak{F}(v) \ni v+\tau \mathfrak{F}(v)$, and using (4.10) in conjunction with (4.16), we obtain

$$
\left\|\mathfrak{F}_{\tau}(v)\right\| \leq\|\mathfrak{F}(v)\| \quad \forall v \in D(\mathfrak{F}) .
$$

\subsection{Explicitly Contractive Operators.}

4.2(a) General Properties. First of all, let us recall that an operator $\mathfrak{H}: \mathscr{B} \rightarrow \mathscr{B}$ is non expansive iff

$$
\|\mathfrak{H}(v)-\mathfrak{H}(w)\| \leq\|v-w\| \quad \forall v, w \in \mathscr{B} ;
$$

it is easy to see that every convex combination of non-expansive operators is non expansive again; moreover if $\mathfrak{H}$ is non-expansive then $I-\mathfrak{H}$ is $m$-accretive (use (4.6b) and (4.4)).

For a fixed $\tau>0$, we say that $\mathfrak{F}$ is a $\tau$-explicitly contractive operator if

$$
D(\mathfrak{F})=\mathscr{B} \quad \text { and } \quad I-\tau \mathfrak{F} \quad \text { is non-expansive. }
$$

Since

$$
I-\lambda \mathfrak{F}=\frac{\lambda}{\tau}(I-\tau \mathfrak{F})+\left(1-\frac{\lambda}{\tau}\right) I, \quad 0 \leq \lambda \leq \tau,
$$

is a convex combination of non-expansive operators, (4.19) is equivalent to $\left(\mathrm{ES}_{2}\right)$ and $\left(\mathrm{ES}_{3}\right)$.

We say that $\mathfrak{F}$ is strongly $\tau$-explicitly contractive if the map

$$
\lambda \mapsto\left\|v_{1}-v_{2}-\lambda\left(\mathfrak{F}\left(v_{1}\right)-\mathfrak{F}\left(v_{2}\right)\right)\right\| \quad \text { is non increasing in }[0, \tau] .
$$

Since the map defined by (4.21) is convex, in fact it is non increasing in $(-\infty, \tau]$. Of course, a strongly $\tau$-explicitly contractive operator is also $\tau$-explicitly contractive. As we already announced in the introduction, we have:

Lemma 4.2 Every $\tau$-explicitly contractive operator $\mathfrak{F}$ is $2 \tau^{-1}$-Lipschitz and satisfies

$$
[\mathfrak{F}(v)-\mathfrak{F}(w), v-w]_{-} \geq 0 \quad \forall v, w \in \mathscr{B} ;
$$

in particular, $\mathfrak{F}$ is $m$-accretive.

Proof. From $\left(\mathrm{ES}_{3}\right)$ we get for every $v_{1}, v_{2} \in \mathscr{B}$,

$\tau\left\|\mathfrak{F}\left(v_{1}\right)-\mathfrak{F}\left(v_{2}\right)\right\| \leq\left\|\left(\tau \mathfrak{F}\left(v_{1}\right)-v_{1}\right)-\left(\tau \mathfrak{F}\left(v_{2}\right)-v_{2}\right)\right\|+\left\|v_{1}-v_{2}\right\| \leq 2\left\|v_{1}-v_{2}\right\|$,

which shows the Lipschitz character of $\mathfrak{F}$. In order to prove (4.22) we simply observe that just by definition

$$
[\mathfrak{F}(v)-\mathfrak{F}(w), v-w]_{-}=\lim _{\lambda \downarrow 0} \frac{\|v-w\|-\|(v-w)-\lambda(\mathfrak{F}(v)-\mathfrak{F}(w))\|}{\lambda} \geq 0,
$$

whence $\mathfrak{F}$ is accretive because of (4.5) and (4.10). Finally, it is well known that every Lipschitz and accretive operator is also $m$-accretive [18, Cor. II.9.2]. 
It is interesting to notice that every Yosida regularization of an $m$-accretive operator is explicitly contractive; in fact, it satisfies the stronger property:

Lemma 4.3 If $\mathfrak{F}$ is $m$-accretive, then $\mathfrak{F}_{\tau}$ is strongly $\tau$-explicitly contractive.

Proof. We already know that $\mathfrak{F}_{\tau}$ is an everywhere defined single-valued operator. Choose $\lambda \leq \tau$ and observe that setting $w_{i}(\lambda):=v_{i}-\lambda \mathfrak{F}_{\tau}\left(v_{i}\right)$ we have

$$
w_{i}(\lambda)=\mathfrak{J}_{\tau}\left(v_{i}\right)+\tau \mathfrak{F}_{\tau}\left(v_{i}\right)-\lambda \mathfrak{F}_{\tau}\left(v_{i}\right)=\mathfrak{J}_{\tau}\left(v_{i}\right)+(\tau-\lambda) \mathfrak{F}_{\tau}\left(v_{i}\right) .
$$

Since $\mathfrak{F}_{\tau}\left(v_{i}\right) \in \mathfrak{F}\left(\mathfrak{J}_{\tau}\left(v_{i}\right)\right)$, by (4.12) we conclude the assertion.

We can revert the previous lemma as follows.

Lemma 4.4 Every strongly $\tau$-explicitly contractive operator $\mathfrak{F}$ can be written (in a unique way) as the $\tau$-Yosida regularization of an $m$-accretive operator $\mathfrak{G}$.

Proof. We define $\mathfrak{G}$ as

$$
\begin{aligned}
& \mathfrak{G}(v):=\mathfrak{F}\left((I-\tau \mathfrak{F})^{-1} v\right)=\{\mathfrak{F}(w): v=w-\tau \mathfrak{F}(w)\}, \\
& D(\mathfrak{G}):=\{v \in \mathscr{B}: v=w-\tau \mathfrak{F}(w) \text { for some } w \in \mathscr{B}\} .
\end{aligned}
$$

Now we check that $\mathfrak{G}$ is accretive: so we fix $v_{1}, v_{2} \in D(\mathfrak{G}), w_{1}, w_{2} \in \mathscr{B}$ with $w_{i}-\tau \mathfrak{F}\left(w_{i}\right)=v_{i}$, and note that

$$
\begin{aligned}
\| v_{1}-v_{2} & +\lambda\left(\mathfrak{G}\left(v_{1}\right)-\mathfrak{G}\left(v_{2}\right)\right) \| \\
& =\left\|w_{1}-\tau \mathfrak{F}\left(w_{1}\right)-\left(w_{2}-\tau \mathfrak{F}\left(w_{2}\right)\right)+\lambda\left(\mathfrak{F}\left(w_{1}\right)-\mathfrak{F}\left(w_{2}\right)\right)\right\| \\
& =\left\|w_{1}-w_{2}+(\lambda-\tau)\left(\mathfrak{F}\left(w_{1}\right)-\mathfrak{F}\left(w_{2}\right)\right)\right\|
\end{aligned}
$$

is a not decreasing function w.r.t. $\lambda$, by definition (4.21). In order to check that $\mathfrak{G}$ is also $m$-accretive, we simply observe that by definition $v:=w-\tau \mathfrak{F}(w)$ is a solution of the equation

$$
v+\tau \mathfrak{G}(v) \ni w, \quad \text { for every choice of } w \in \mathscr{B} .
$$

Finally, if $v$ is the (unique) solution of (4.26),

$$
\mathfrak{G}_{\tau}(w)=\frac{w-v}{\tau}=\frac{w-(w-\tau \mathfrak{F}(w))}{\tau}=\mathfrak{F}(w),
$$

as asserted.

As a last result for these class of operators, we present a useful characterization of strongly $\tau$-explicitly contractive operators in Hilbert spaces, which is intimately related to a coercivity type property.

Proposition 4.1 Let $\mathscr{B}$ be a Hilbert space with scalar product $\langle\cdot, \cdot\rangle$. Then $\mathfrak{F}$ is a strongly $\tau$-explicitly contractive operator iff

$$
\left\langle\mathfrak{F}\left(v_{1}\right)-\mathfrak{F}\left(v_{2}\right), v_{1}-v_{2}\right\rangle \geq \tau\left\|\mathfrak{F}\left(v_{1}\right)-\mathfrak{F}\left(v_{2}\right)\right\|^{2}, \quad \forall v_{1}, v_{2} \in \mathscr{B} .
$$


Proof. We simply take the derivative of the (square of the) map (4.21)

$$
\begin{aligned}
0 & \geq \frac{d}{d \lambda}\left\|v_{1}-v_{2}-\lambda\left(\mathfrak{F}\left(v_{1}\right)-\mathfrak{F}\left(v_{2}\right)\right)\right\|^{2}= \\
& =-2\left\langle\mathfrak{F}\left(v_{1}\right)-\mathfrak{F}\left(v_{2}\right), v_{1}-v_{2}-\lambda\left(\mathfrak{F}\left(v_{1}\right)-\mathfrak{F}\left(v_{2}\right)\right)\right\rangle, \quad \forall \lambda \in[0, \tau],
\end{aligned}
$$

and we deduce (4.27) upon choosing $\lambda=\tau$. The converse is trivial from the previous equality.

Corollary 4.5 Let $\mathscr{B}$ be a Hilbert space with scalar product $\langle\cdot, \cdot\rangle$. Then $\mathfrak{F}$ is a $\tau$-explicitly contractive operator iff it is strongly $\tau / 2$-explicitly contractive.

Proof. $\quad \mathfrak{F}$ is a $\tau$-explicitly contractive operator iff

$$
\left\|v_{1}-v_{2}-\tau\left(\mathfrak{F}\left(v_{1}\right)-\mathfrak{F}\left(v_{2}\right)\right)\right\|^{2} \leq\left\|v_{1}-v_{2}\right\|^{2} \quad \forall v_{1}, v_{2} \in \mathscr{B} .
$$

Since $\mathscr{B}$ is a Hilbert space, the previous inequality is equivalent to

$$
-2 \tau\left\langle v_{1}-v_{2}, \mathfrak{F}\left(v_{1}\right)-\mathfrak{F}\left(v_{2}\right)\right\rangle+\tau^{2}\left\|\mathfrak{F}\left(v_{1}\right)-\mathfrak{F}\left(v_{2}\right)\right\|^{2} \leq 0,
$$

i.e.

$$
\left\langle v_{1}-v_{2}, \mathfrak{F}\left(v_{1}\right)-\mathfrak{F}\left(v_{2}\right)\right\rangle \geq \frac{\tau}{2}\left\|\mathfrak{F}\left(v_{1}\right)-\mathfrak{F}\left(v_{2}\right)\right\|^{2} .
$$

Applying Proposition 4.1, we deduce the assertion.

Remark 4.6 In view of Lemmas 4.3, 4.4 and Corollary 4.5, the class of explicitly contractive operators in a Hilbert space coincides with the class of Yosida regularizations up to a factor 2 in the parameters.

4.2(b) Explicitly Contractive Operators w.r.t. $L^{1}$ and $L^{\infty}$ Norms. Proposition 4.1 gives a direct characterization of explicitly contractive operators in the case of an Hilbert norm. Now we want to show other useful characterizations in the case of norms of $L^{1}, L^{\infty}$-type.

First of all we recall a crucial result of CRANDALL and TARTAR [17].

Lemma 4.7 Let $(\Omega, \mathscr{S}, \mu)$ be a measure space.

1. If $\mathfrak{H}: L^{1}(\Omega, \mathscr{S}, \mu) \rightarrow L^{1}(\Omega, \mathscr{S}, \mu)$ is an operator satisfying

$$
\int_{\Omega} \mathfrak{H}(u) d \mu=\int_{\Omega} u d \mu \quad \forall u \in L^{1}(\Omega, \mathscr{S}, \mu) .
$$

then it is non expansive (4.18) if and only if it is order preserving, i.e.

$$
u \leq v \mu \text {-a.e. in } \Omega \Rightarrow \mathfrak{H}(u) \leq \mathfrak{H}(v) \mu \text {-a.e. in } \Omega \text {. }
$$

2. If $\mathfrak{H}: L^{\infty}(\Omega, \mathscr{S}, \mu) \rightarrow L^{\infty}(\Omega, \mathscr{S}, \mu)$ is an operator satisfying

$$
\mathfrak{H}(u+c)=\mathfrak{H}(u)+c \quad \forall u \in L^{\infty}(\Omega, \mathscr{S}, \mu), c \in \mathbb{R},
$$

then it is non expansive (4.18) if and only if it is order preserving (4.29).

Remark 4.8 In the second statement of the previous lemma it is always possible to replace $L^{\infty}(\Omega, \mathscr{S}, \mu)$ by $B(\Omega)$ (the space of the bounded real functions defined on $\Omega$ with the "sup" norm) or by any closed subspace containing the constant functions. In this case no measures are involved and the order preserving property (4.29) should be intended pointwise everywhere. 
Corollary 4.9 Let $\mathfrak{F}: \mathbb{R}^{d} \rightarrow \mathbb{R}^{d}$ be a given locally Lipschitz map with components $\mathfrak{F}_{1}, \ldots, \mathfrak{F}_{d}: \mathbb{R}^{d} \rightarrow \mathbb{R}$, and let $\alpha_{1}, \ldots, \alpha_{d}$ be given positive numbers; we denote by $\|\cdot\|_{1},\|\cdot\|_{\infty}$ respectively the norms in $\mathbb{R}^{d}$ defined by

$$
\|v\|_{1}:=\sum_{i=1}^{d} \alpha_{i}\left|v_{i}\right|, \quad\|v\|_{\infty}:=\sup _{1 \leq i \leq d} \alpha_{i}\left|v_{i}\right| \quad \forall v=\left(v_{1}, \ldots, v_{d}\right) \in \mathbb{R}^{d} .
$$

1. Suppose that

$$
\sum_{i=1}^{d} \alpha_{i} \mathfrak{F}_{i}(v)=0 \quad \forall v \in \mathbb{R}^{d} .
$$

Then $\mathfrak{F}$ is explicitly $\tau$-contractive w.r.t. the $\|\cdot\|_{1}$-norm if and only if

$$
\begin{gathered}
\frac{\partial \mathfrak{F}_{i}}{\partial x_{j}} \leq 0 \quad \text { a.e. in } \mathbb{R}^{d}, \text { for } 1 \leq i \neq j \leq d \\
0 \leq \frac{\partial \mathfrak{F}_{i}}{\partial x_{i}} \leq \frac{1}{\tau} \quad \text { a.e. in } \mathbb{R}^{d}, \text { for } 1 \leq i \leq d .
\end{gathered}
$$

2. Suppose that

$$
\sum_{j=1}^{d} \frac{\partial \mathfrak{F}_{i}}{\partial x_{j}}=0 \quad \text { a.e. in } \mathbb{R}^{d}, \text { for } i=1, \ldots, d,
$$

or, equivalently, that for every choice of $x=\left(x_{1}, x_{2}, \ldots, x_{d}\right) \in \mathbb{R}^{d}$ and $i=1, \ldots, d$

$$
\mathfrak{F}_{i}\left(x_{1}+c, x_{2}+c, \ldots, x_{d}+c\right)=\mathfrak{F}_{i}\left(x_{1}, x_{2}, \ldots, x_{d}\right) \quad \forall c \in \mathbb{R} .
$$

Then $\mathfrak{F}$ is explicitly $\tau$-contractive w.r.t. the $\|\cdot\|_{\infty}$-norm if and only if (4.33) holds.

\subsection{Properties of Integral Solutions.}

4.3(a) Inequalities in the Sense of Distributions. We recall here a definition which we will extensively use in the following.

If $\zeta \in C^{0}(0, T), \bar{\zeta} \in L^{1}(0, T)$, then we say that

$$
\left\{\begin{array}{cl}
\frac{d}{d t} \zeta+\bar{\zeta} \leq 0 \quad \text { in } \mathscr{D}^{\prime}(0, T) & \text { if and only if } \\
\zeta(\beta)-\zeta(\alpha)+\int_{\alpha}^{\beta} \bar{\zeta}(t) d t \leq 0 \quad & \forall 0 \leq \alpha \leq \beta \leq T .
\end{array}\right.
$$

Observe that if $\zeta$ is absolutely continuous, i.e. $\zeta \in W^{1,1}(0, T)$, then (4.37) is also equivalent to the more familiar

$$
\frac{d}{d t} \zeta(t)+\bar{\zeta}(t) \leq 0 \quad \text { for a.e. } t \in(0, T) .
$$


4.3(b) Functions of Bounded Variation. We denote by $B V(0, T ; \mathscr{B})$ the Banach space of functions $f:[0, T] \rightarrow \mathscr{B}$ such that

$$
\operatorname{Var} f:=\sup _{0 \leq r_{0}<r_{1}<\ldots<r_{J} \leq T} \sum_{j=1}^{J}\left\|f\left(r_{j}\right)-f\left(r_{j-1}\right)\right\|<+\infty
$$

with the norm

$$
\|f\|_{B V(0, T ; \mathscr{B})}:=\|f(0)\|+\operatorname{Var} f .
$$

By extending $f(t):=f(0)$ to $(-\infty, 0)$, it is not difficult to see that [7, Appendix]

$$
\begin{aligned}
\int_{0}^{h}\|f(t)-f(0)\| d t & +\int_{h}^{T}\|f(t)-f(t-h)\| d t \\
& =\int_{0}^{T}\|f(t)-f(t-h)\| d t \leq h \operatorname{Var} f \quad \forall h \in[0, T] .
\end{aligned}
$$

4.3(c) Evolution Equations: Strong and Integral Solutions. Let $\mathfrak{F}$ be an accretive operator in $\mathscr{B}$. Given

$$
\text { an initial datum } u_{0} \in \overline{D(\mathfrak{F})} \text { and a function } f \in L^{1}(0, T ; \mathscr{B}) \text {, }
$$

we say that $u \in C^{0}([0, T] ; \mathscr{B})$ is a strong solution of the Cauchy problem

$$
\left\{\begin{array}{l}
u^{\prime}+\mathfrak{F}(u) \ni f(t) \\
u(0)=u_{0}
\end{array}\right.
$$

if $u$ is also absolutely continuous in $[0, T]$ and it satisfies (CP) at almost every point; in particular $u(t) \in D(\mathfrak{F})$ for a.e. $t \in(0, T)$.

It is possible to write an integral formulation of $(\mathrm{CP})$ which will turn out to be useful: since $u^{\prime}(t)=f(t)-\xi(t), \xi(t) \in \mathfrak{F}(u(t))$, by (4.7) we get for all $v \in \mathscr{B}$

$$
\frac{d}{d t}\|u(t)-v\|=\left[u^{\prime}(t), u(t)-v\right]_{-}=[f(t)-\xi(t), u(t)-v]_{-} \text {a.e. in }(0, T) .
$$

Therefore, in view of (4.3), if $u$ is a strong solution then

$$
\frac{d}{d t}\|u(t)-v\|+[\mathfrak{F}(u(t))-f(t), u(t)-v]_{+} \leq 0, \text { a.e. in }(0, T), \forall v \in \mathscr{B},
$$

where $\mathfrak{F}(u(t))$ denotes the $L^{1}(0, T ; \mathscr{B})$ selection $\xi(t)$ introduced before. If $v \in$ $D(\mathfrak{F})$, by $(4.3),(4.6 \mathrm{c})$ and (4.10) this last formula yields

$$
\frac{d}{d t}\|u(t)-v\|+[\mathfrak{F}(v)-f(t), u(t)-v]_{-} \leq 0, \quad \forall v \in D(\mathfrak{F}) .
$$

We say that $u \in C^{0}([0, T] ; \mathscr{B})$ is a weak integral solution (in the sense of BÉNILAN [4]) if it satisfies (4.45) in the sense of distributions (4.37).

It is clear that strong solutions are also integral solutions. Conversely, if $\mathfrak{F}$ is maximal accretive and $u$ is a differentiable integral solution, then $u$ is also a strong solution. 
4.3(d) Existence, Uniqueness and Regularity Results [15, 4, 12]. If $\mathfrak{F}$ is $m$-accretive, then for every $u_{0} \in \overline{D(\mathfrak{F})}$ and $f \in L^{1}(0, T ; \mathscr{B})$ there exists a unique weak integral solution $u$ of $(\mathrm{CP})$. The map $\left(u_{0}, f\right) \mapsto u$ is non-expansive, i.e. if $v$ is the integral solution w.r.t. $v_{0}, g$ then

$$
\sup _{t \in[0, T]}\|u(t)-v(t)\| \leq\left\|u_{0}-v_{0}\right\|+\|f-g\|_{L^{1}(0, T ; \mathscr{B})} .
$$

Moreover if $u_{0} \in D(\mathfrak{F})$ and $f \in B V(0, T ; \mathscr{B})$ then we introduce the following measure of regularity and compatibility of data

$$
\rho\left(u_{0}, f\right):=\inf _{\hat{z} \in \mathfrak{F}\left(u_{0}\right)}(\|f(0)-\hat{z}\|+\operatorname{Var} f) \quad \forall u_{0} \in D(\mathfrak{F}), f \in B V(0, T ; \mathscr{B}),
$$

and we realize that $u$ is also Lipschitz continuous with values in $\mathscr{B}$ (see also [30, Chap. IV, §8], [24, Chap. 5, §4], [2, Chap. III, § 2]) and

$$
\|u(t)-u(s)\| \leq|t-s| \rho\left(u_{0}, f\right) \quad \forall s, t \in[0, T] .
$$

Consequently (4.46) and (4.48) suggest a simple way to estimate the modulus of continuity of a general integral solution $u$ of $(\mathrm{CP})$ in terms of the data. We introduce the following definition which is intimately related to the Peetre's K-functional [5] (see also [6] for a similar setting).

Definition 4.10 (Modulus of Regularity) For all $v \in \mathscr{B}, f \in L^{1}(0, T ; \mathscr{B})$, the modulus of regularity $\omega(\cdot ; v, f)$ is the (non-decreasing, concave) function defined in the interval $[0,+\infty)$ by

$$
\begin{gathered}
\omega(\varepsilon ; v, f):=\inf \left\{\|v-z\|+\|f-\psi\|_{L^{1}(0, T ; \mathscr{B})}+\varepsilon \rho(z, \psi):\right. \\
z \in D(\mathfrak{F}), \psi \in B V(0, T ; \mathscr{B})\} .
\end{gathered}
$$

A standard density argument shows that

$$
u_{0} \in \overline{D(\mathfrak{F})}, f \in L^{1}(0, T ; \mathscr{B}) \quad \Rightarrow \quad \lim _{\varepsilon \downarrow 0} \omega\left(\varepsilon ; u_{0}, f\right)=0,
$$

whereas, taking $z=u_{0}, \psi=f$ one gets immediately

$$
u_{0} \in D(\mathfrak{F}), f \in B V(0, T ; \mathscr{B}) \quad \Rightarrow \quad \omega\left(\varepsilon ; u_{0}, f\right) \leq \varepsilon \rho\left(u_{0}, f\right) .
$$

Combining (4.46) and (4.48) it is easy to see that the modulus of continuity of the integral solution $u$ of $(\mathrm{CP})$ w.r.t. $u_{0}, f$ can be estimated by

$$
|t-s| \leq \varepsilon \quad \Rightarrow \quad\|u(t)-u(s)\| \leq 2 \omega\left(\varepsilon / 2 ; u_{0}, f\right) .
$$

4.3(e) Piecewise Constant Functions. Let us denote by $\mathbb{P}^{0}(\mathcal{P})$ the space of piecewise constant functions on the grid $\mathcal{P}$ of (1.1)

$$
\mathbb{P}^{0}(\mathcal{P}):=\left\{\bar{\Psi}:[0, T] \rightarrow \mathscr{B}: \bar{\Psi} \equiv \Psi_{n} \text { if } t \in\left(t_{n-1}, t_{n}\right], n=1, \ldots N\right\} .
$$

There is a natural projection operator $\Pi_{\mathcal{P}}^{0}: L^{1}(0, T ; \mathscr{B}) \rightarrow \mathbb{P}^{0}(\mathcal{P})$ defined as

$$
\bar{\Psi}=\Pi_{\mathcal{P}}^{0} \psi \Leftrightarrow \Psi_{n}=f_{t_{n-1}}^{t_{n}} \psi(t) d t
$$


which is non-expansive w.r.t. the $L^{1}(0, T ; \mathscr{B})$-norm and variation diminishing, when we choose $\Psi_{0}=\bar{\Psi}(0)=\psi(0)$

$$
\bar{\Psi}=\Pi_{\mathcal{P}}^{0} \psi \Rightarrow \operatorname{Var} \bar{\Psi} \leq \operatorname{Var} \psi .
$$

Therefore, in case $f=\bar{F} \in \mathbb{P}^{0}(\mathcal{P})$ it is easy to check that the computation of $\omega(\cdot ; v, \bar{F})$ just invokes a minimization on a finite number of variables in $\mathscr{B}$ :

$$
\begin{gathered}
\omega(\varepsilon ; v, \bar{F}):=\inf \left\{\|v-z\|+\|\bar{F}-\bar{\Psi}\|_{L^{1}(0, T ; \mathscr{B})}+\varepsilon \rho(z, \bar{\Psi}):\right. \\
\left.z \in D(\mathfrak{F}), \bar{\Psi} \in \mathbb{P}^{0}(\mathcal{P})\right\} .
\end{gathered}
$$

\section{Relaxed Solutions: Motivation}

In this section we exploit $\left(\mathrm{IS}_{1}\right)$ and $\left(\mathrm{ES}_{1}\right)$ to construct relaxed solutions to $(\mathrm{CP})$.

We recall (cf. (1.4), (1.5)) that $U(t)$ is the piecewise linear interpolant of the values $\left\{U_{n}\right\}_{n=0}^{N}$ on the grid $\mathcal{P}=\left\{t_{n}\right\}_{n=0}^{N}$ of (1.1), and that $\bar{U}(t), \bar{F}(t)$ are the piecewise constant functions which respectively take the values $U_{n}, F_{n}$ in the interval $\left(t_{n-1}, t_{n}\right]$.

\section{$5.1 \quad$ Implicit Euler Scheme.}

We first deal with the implicit method $\left(\mathrm{IS}_{1}\right)$.

Theorem 2 Let $\left\{U_{n}\right\}_{n=0}^{N}$ be the discrete solution of the Euler implicit scheme $\left(\mathrm{IS}_{1,2,3}\right)$ and let $U, \bar{U}, \bar{F}$ be defined in (1.4) and (1.5). Then

$$
\frac{d}{d t}\|U(t)-w\|+[\mathfrak{F}(\bar{U}(t))-\bar{F}(t), \bar{U}(t)-w]_{+} \leq 0 \quad \text { in } \mathscr{D}^{\prime}(0, T), \quad \forall w \in \mathscr{B},
$$

where $\mathfrak{F}(\bar{U}(t))$ denotes the $L^{1}(0, T ; \mathscr{B})$ selection $\xi(t):=\bar{F}(t)-U^{\prime}(t)$.

Proof. Since $U(t)$ and $\|U(t)-w\|$ are piecewise $C^{1}$ functions, we will show that (5.1) holds a.e. in $(0, T)$. Let us suppose that $t \in\left(t_{n-1}, t_{n}\right]$ for a fixed $n$ between 1 and $N$. Since

$$
U^{\prime}(t) \equiv \frac{U_{n}-U_{n-1}}{\tau_{n}} \quad \forall t \in\left(t_{n-1}, t_{n}\right)
$$

we deduce that $U, \bar{U}$ satisfy

$$
U^{\prime}(t)+\mathfrak{F}(\bar{U}(t)) \ni \bar{F}(t) \quad \forall t \in\left(t_{n-1}, t_{n}\right) .
$$

Moreover, since $U(t)$ is linear, for every $w \in \mathscr{B}$ the map

$$
t \mapsto\|U(t)-w\| \quad \text { is convex in }\left[t_{n-1}, t_{n}\right] ;
$$

in particular its time derivative is nondecreasing and is bounded above by the left derivative at $t:=t_{n}$. Therefore, in view of $(4.2 \mathrm{~b})$, we have for a.e. $t \in\left(t_{n-1}, t_{n}\right]$

$$
\begin{aligned}
\frac{d}{d t}\|U(t)-w\| & \leq \lim _{\lambda \downarrow 0} \frac{\left\|U\left(t_{n}\right)-w\right\|-\left\|U\left(t_{n}-\lambda\right)-w\right\|}{\lambda} \\
& =\lim _{\lambda \downarrow 0} \frac{\left\|U\left(t_{n}\right)-w\right\|-\left\|U\left(t_{n}\right)-w-\lambda U^{\prime}(t)\right\|}{\lambda} \\
& =\left[U^{\prime}(t), U\left(t_{n}\right)-w\right]_{-}=\left[U^{\prime}(t), \bar{U}(t)-w\right]_{-} .
\end{aligned}
$$


Taking (5.2) into account and setting $\xi(t):=\bar{F}(t)-U^{\prime}(t) \in \mathfrak{F}(\bar{U}(t))$, we get

$$
\begin{aligned}
\frac{d}{d t}\|U(t)-w\| & +[\xi(t)-\bar{F}(t), \bar{U}(t)-w]_{+} \\
\leq & {\left[U^{\prime}(t), \bar{U}(t)-w\right]_{-}+\left[-U^{\prime}(t), \bar{U}(t)-w\right]_{+}=0, }
\end{aligned}
$$

where we have used (4.3). We thus conclude that the pair $(U, \bar{U})$ satisfies the dissipation inequality (5.1).

\subsection{Explicit Euler Scheme.}

We now consider the explicit method $\left(\mathrm{ES}_{1}\right)$ and we introduce the auxiliary piecewise linear function $\tilde{U}(t, w)$ interpolating the discrete values $\left\{\left\|U_{n}-w\right\|\right\}_{n=0}^{N}$ for every $w \in \mathscr{B}$; thus for every $w \in \mathscr{B}$

$$
\tilde{U}(t, w):=\ell(t)\left\|U_{n}-w\right\|+(1-\ell(t))\left\|U_{n-1}-w\right\|, \quad \forall t \in\left[t_{n-1}, t_{n}\right],
$$

where

$$
\ell(t):=\frac{t-t_{n-1}}{\tau_{n}}, \quad \forall t \in\left(t_{n-1}, t_{n}\right]
$$

The reader could compare this approach with the analogous one adopted in $[1$, Chap. 5].

Theorem 3 Let $\left\{U_{n}\right\}_{n=0}^{N}$ be the discrete solution of the explicit Euler scheme $\left(\mathrm{ES}_{1,2,3}\right)$ and let $\tilde{U}, \bar{U}, \bar{F}$ be defined in (5.4) and (1.5). For every choice of $w \in D(\mathfrak{F})$

$$
\frac{d}{d t} \tilde{U}(t, w)+[\mathfrak{F}(w)-\bar{F}(t), \bar{U}(t)-w]_{+} \leq 0 \quad \text { in } \mathscr{D}^{\prime}(0, T) .
$$

Proof. ¿From $\left(\mathrm{ES}_{1}\right)$ we deduce

$$
U_{n}=U_{n-1}-\tau_{n} \mathfrak{F}\left(U_{n-1}\right)+\tau_{n} F_{n} .
$$

Since $\left(\mathrm{ES}_{3}\right)$ yields

$$
\left\|\left(U_{n-1}-\tau_{n} \mathfrak{F}\left(U_{n-1}\right)\right)-\left(w-\tau_{n} \mathfrak{F}(w)\right)\right\| \leq\left\|U_{n-1}-w\right\| \quad \forall w \in \mathscr{B},
$$

with the aid of (4.2a) and (5.7), we can write

$$
\begin{aligned}
\left\|U_{n}-w\right\|-\left\|U_{n-1}-w\right\| & \leq\left\|U_{n}-w\right\|-\left\|U_{n}-w+\tau_{n}\left(\mathfrak{F}(w)-F_{n}\right)\right\| \\
& \leq-\tau_{n}\left[\mathfrak{F}(w)-F_{n}, U_{n}-w\right]_{+} .
\end{aligned}
$$

We have thus arrived at

$$
\left\|U_{n}-w\right\|-\left\|U_{n-1}-w\right\|+\tau_{n}\left[\mathfrak{F}(w)-F_{n}, U_{n}-w\right]_{+} \leq 0,
$$

which can be equivalently written as the dissipation inequality (5.6). 


\subsection{Yosida Regularization.}

For $\tau>0$ let $\mathfrak{J}_{\tau}, \mathfrak{F}_{\tau}$ be the resolvent and the Yosida regularization of $\mathfrak{F}$ introduced in (4.15) and (4.16). For every $u_{0} \in \mathscr{B}, f \in L^{1}(0, T ; \mathscr{B})$ CauchyLipschitz-Picard theorem [8, Theorem VII.3] entails the existence of the strong $W^{1,1}([0, T] ; \mathscr{B})$ solution $u_{\tau}$ of

$$
\left\{\begin{array}{l}
u_{\tau}^{\prime}(t)+\mathfrak{F}_{\tau}\left(u_{\tau}(t)\right)=f(t), \quad t \in[0, T] \\
u_{\tau}(0)=u_{0} .
\end{array}\right.
$$

To prove a dissipation inequality for $u_{\tau}$, we introduce the auxiliary functions

$$
f_{\tau}(t):=\frac{1}{\tau} \int_{0}^{t} e^{(s-t) / \tau} f(s) d s, \quad \text { solutions of }\left\{\begin{aligned}
\tau f_{\tau}^{\prime}(t)+f_{\tau}(t) & =f(t), \\
f_{\tau}(0) & =0 .
\end{aligned}\right.
$$

Theorem 4 The functions

$$
\tilde{u}_{\tau}(t, w):=\left\|u_{\tau}(t)-\tau f_{\tau}(t)-w\right\|, \quad \bar{u}_{\tau}(t):=\mathfrak{J}_{\tau}\left(u_{\tau}(t)\right)
$$

satisfy the dissipation inequality

$$
\frac{d}{d t} \tilde{u}_{\tau}(t, w)+\left[\mathfrak{F}\left(\bar{u}_{\tau}(t)\right)-f_{\tau}(t), \bar{u}_{\tau}(t)-w\right]_{+} \leq 0 \quad \text { in } \mathscr{D}^{\prime}(0, T),
$$

where $\mathfrak{F}\left(\bar{u}_{\tau}(t)\right) \in L^{1}(0, T ; \mathscr{B})$ denotes the selection $\xi(t):=\mathfrak{F}_{\tau}\left(u_{\tau}(t)\right) \in \mathfrak{F}\left(\bar{u}_{\tau}(t)\right)$.

Proof. In view of (5.8) and (5.9) we have

$$
\left.\frac{d}{d t}\left(u_{\tau}(t)-\tau f_{\tau}(t)\right)+\mathfrak{F}_{\tau}\left(u_{\tau}(t)\right)=f_{\tau}(t) \quad \forall t \in\right] 0, T[,
$$

whence, invoking (4.7) and (4.16),

$$
\begin{aligned}
\frac{d}{d t} \tilde{u}_{\tau}(t, w) & =\left[\frac{d}{d t}\left(u_{\tau}(t)-\tau f_{\tau}(t)\right), u_{\tau}(t)-\tau f_{\tau}(t)-w\right]_{-} \\
& =\left[f_{\tau}-\mathfrak{F}_{\tau}\left(u_{\tau}\right), u_{\tau}-\tau f_{\tau}-w\right]_{-} \\
& =\left[f_{\tau}-\frac{u_{\tau}-\bar{u}_{\tau}}{\tau}, u_{\tau}-\tau f_{\tau}-w\right]_{-} \\
& =\frac{1}{\tau}\left[\bar{u}_{\tau}-w-\left(u_{\tau}-\tau f_{\tau}-w\right), u_{\tau}-\tau f_{\tau}-w\right]_{-} \\
& \leq \frac{1}{\tau}\left[\bar{u}_{\tau}-w-\left(u_{\tau}-\tau f_{\tau}-w\right), \bar{u}_{\tau}-w\right]_{-} \\
& =\left[f_{\tau}-\mathfrak{F}_{\tau}\left(u_{\tau}\right), \bar{u}_{\tau}-w\right]_{-},
\end{aligned}
$$

which implies (5.11). In the last inequality, we used the monotonicity property

$$
[v-w, w]_{-} \leq[v-w, v]_{-} \quad \forall v, w \in \mathscr{B},
$$

which follows directly from $(4.6 \mathrm{a}, \mathrm{b}, \mathrm{c})$. This completes the proof.

Remark 5.1 Observe that in the homogeneous case $f \equiv 0$, equations (5.9) and (5.10) reduce to the considerably simpler form

$$
f_{\tau}(t) \equiv 0, \quad \tilde{u}_{\tau}(t, w):=\left\|u_{\tau}(t)-w\right\|, \quad \bar{u}_{\tau}(t)=\mathfrak{J}_{\tau}\left(u_{\tau}(t)\right) .
$$




\section{Relaxed Solutions: Definition and Compari- son Principle}

The novel concept of relaxed solution of the Cauchy problem (CP)

$$
\left\{\begin{array}{l}
u^{\prime}+\mathfrak{F}(u) \ni f(t) \\
u(0)=u_{0}
\end{array}\right.
$$

for an accretive operator $\mathfrak{F}$ is now introduced and fully discussed. This concept is inspired in (5.1), (5.6), and (5.11).

Definition 6.1 (Relaxed solutions) We say that a couple of functions

$$
\boldsymbol{u}:=(\tilde{u}, \bar{u}) \in C^{0}([0, T] \times \mathscr{B} ; \mathbb{R}) \times L^{1}(0, T ; \mathscr{B})
$$

is a

$$
\text { strong relaxed solution of }(\mathrm{CP}) \text { w.r.t. } u_{0} \in \mathscr{B}, f \in L^{1}(0, T ; \mathscr{B})
$$

if (there exists a suitable selection $\xi(t) \in L^{1}(0, T ; \mathscr{B})$ of $\mathfrak{F}(\bar{u}(t))$ s.t.)

$$
\begin{gathered}
\frac{d}{d t} \tilde{u}(t, w)+[\xi(t)-f(t), \bar{u}(t)-w]_{+} \leq 0 \quad \text { in } \mathscr{D}^{\prime}(0, T), \quad \forall w \in \mathscr{B}, \\
\tilde{u}(0, w) \leq\left\|u_{0}-w\right\| \quad \forall w \in \mathscr{B} .
\end{gathered}
$$

We say that $\boldsymbol{u}=(\tilde{u}, \bar{u})$ as in (6.1) is a weak relaxed solution of (CP) w.r.t. $u_{0} \in \mathscr{B}, f \in L^{1}(0, T ; \mathscr{B})$ if

$$
\begin{gathered}
\frac{d}{d t} \tilde{u}(t, w)+[\zeta-f(t), \bar{u}(t)-w]_{-} \leq 0 \quad \text { in } \mathscr{D}^{\prime}(0, T), \quad \forall w \in D(\mathfrak{F}), \\
\tilde{u}(0, w) \leq\left\|u_{0}-w\right\| \quad \forall w \in D(\mathfrak{F}),
\end{gathered}
$$

for all $\zeta \in \mathfrak{F}(w)$. The discrepancy of a relaxed solution is defined by

$$
\Delta_{\boldsymbol{u}}:=\int_{0}^{T} \delta_{\boldsymbol{u}}(t) d t
$$

where, for a.e. $t \in(0, T), \delta_{\boldsymbol{u}}(t):=\sup _{w \in \mathscr{B}}|\tilde{u}(t, w)-\|\bar{u}(t)-w\||$.

The deviation of two relaxed solutions $\boldsymbol{u}, \boldsymbol{v}$ at the time $t \in[0, T]$ is defined by

$$
\mathfrak{D}(t ; \boldsymbol{u}, \boldsymbol{v}):=\inf _{w \in \mathscr{B}}(\tilde{u}(t, w)+\tilde{v}(t, w)) .
$$

Example 6.2 (Implicit Euler Scheme) In view of Theorem 2, the pair $\boldsymbol{u}:=$ $(\tilde{U}, \bar{U})$ with $\tilde{U}=\|U-w\|$ is a strong relaxed solution w.r.t. $U_{0}, \bar{F}$ of $(\mathrm{CP})$, and

$$
\begin{gathered}
\delta_{\boldsymbol{u}}(t)=\frac{t_{n}-t}{\tau_{n}}\left\|U_{n}-U_{n-1}\right\| \quad \forall t_{n-1}<t \leq t_{n}, \\
\Delta_{\boldsymbol{u}}=\frac{1}{2} \sum_{n=1}^{N} \tau_{n}\left\|U_{n}-U_{n-1}\right\| .
\end{gathered}
$$


Example 6.3 (Explicit Euler Scheme) In view of Theorem 3, the pair $\boldsymbol{u}:=$ $(\tilde{U}, \bar{U})$ is a weak relaxed solution w.r.t. $U_{0}, \bar{F}$ of $(\mathrm{CP})$. Compared with Example 6.2 , where $\tilde{U}(t, w)$ is the norm of the piecewise linear function $U(t)-w$, now $\tilde{U}(t, w)$ is the piecewise linear interpolant of the norms $\left\{\left\|U_{n}-w\right\|\right\}_{n=0}^{N}$ :

$$
\tilde{U}(t, w)=\ell(t)\left\|U_{n}-w\right\|+(1-\ell(t))\left\|U_{n-1}-w\right\| .
$$

Using this expression, it is easy to see that $\delta_{\boldsymbol{u}}(t)$ and $\Delta_{\boldsymbol{u}}$ also satisfy (6.9).

Example 6.4 (Yosida Regularization) In view of Theorem 4, the pair $\boldsymbol{u}:=$ $\left(\tilde{u}_{\tau}, \bar{u}_{\tau}\right)$ defined in (5.10) is a strong relaxed solution w.r.t. $u_{0}, f_{\tau}$ of $(\mathrm{CP})$ with

$$
\begin{gathered}
\delta_{\boldsymbol{u}}(t)=\left\|u_{\tau}-\tau f_{\tau}-\mathfrak{J}_{\tau}\left(u_{\tau}\right)\right\|=\tau\left\|\mathfrak{F}_{\tau}\left(u_{\tau}\right)-f_{\tau}\right\|, \\
\Delta_{\boldsymbol{u}}=\tau \operatorname{Var}\left(u_{\tau}-\tau f_{\tau}\right),
\end{gathered}
$$

because

$$
\mathfrak{F}_{\tau}\left(u_{\tau}\right)-f_{\tau}=\mathfrak{F}_{\tau}\left(u_{\tau}\right)-f+f-f_{\tau}=-u_{\tau}^{\prime}+\tau f_{\tau}^{\prime} .
$$

Remark 6.5 A strong relaxed solution is also a weak relaxed solution. Notice that $u$ is a strong (or integral) solution of $(\mathrm{CP})$, as defined in $\S 4.3(c)$, if and only if the couple

$$
\tilde{u}(t, w):=\|u(t)-w\|, \quad \bar{u}(t):=u(t),
$$

is also a relaxed strong (respectively, weak) solution of the same equation with discrepancy $\Delta_{u}=0$.

Remark 6.6 Observe that $\mathfrak{D}(t ; \boldsymbol{u}, \boldsymbol{v})$, together with $\delta_{\boldsymbol{u}}(t), \delta_{\boldsymbol{v}}(t)$, provide control of $\|\bar{u}-\bar{v}\|$. In fact, for every $z \in \mathscr{B}$

$$
\|\bar{u}(t)-\bar{v}(t)\| \leq\|\bar{u}(t)-z\|-\tilde{u}(t, z)+\tilde{u}(t, z)+\tilde{v}(t, z)+\|z-\bar{v}(t)\|-\tilde{v}(t, z),
$$

whence

$$
\|\bar{u}(t)-\bar{v}(t)\| \leq \delta_{\boldsymbol{u}}(t)+\mathfrak{D}(t ; \boldsymbol{u}, \boldsymbol{v})+\delta_{\boldsymbol{v}}(t) .
$$

In particular, we have the $L^{1}$-error bound

$$
\|\bar{u}-\bar{v}\|_{L^{1}(0, T ; \mathscr{B})} \leq \Delta_{\boldsymbol{u}}+\Delta_{\boldsymbol{v}}+\int_{0}^{T} \mathfrak{D}(t ; \boldsymbol{u}, \boldsymbol{v}) d t .
$$

Theorem 5 Let us assume that

$$
\begin{aligned}
\boldsymbol{u} & :=(\tilde{u}, \bar{u}) \text { is a strong relaxed solution w.r.t. } u_{0}, f \text { in the interval }(0, T), \\
\boldsymbol{v} & :=(\tilde{v}, \bar{v}) \text { is a weak relaxed solution w.r.t. } v_{0}, g \text { in the interval }(0, T) .
\end{aligned}
$$

Then, the deviation $\mathfrak{D}(T ; \boldsymbol{u}, \boldsymbol{v})$ of $\boldsymbol{u}$ and $\boldsymbol{v}$ at time $T$ satisfies

$$
\mathfrak{D}(T ; \boldsymbol{u}, \boldsymbol{v}) \leq\left\|u_{0}-v_{0}\right\|+\|f-g\|_{L^{1}(0, T ; \mathscr{B})}+4 \Omega\left(\Delta_{\boldsymbol{u}}+\frac{1}{2} \Delta_{\boldsymbol{v}} ; u_{0}, f\right),
$$

where the regularity function $\Omega$ associated with $u_{0}, f$ is defined as follows in terms of the modulus of regularity $\omega$ of Definition 4.10:

$$
\Omega\left(\delta ; u_{0}, f\right):=\inf _{\varepsilon>0}\left(\omega\left(\varepsilon / 2 ; u_{0}, f\right)+\frac{\delta}{2 \varepsilon}\right) \quad \forall \delta \geq 0 .
$$


Remark 6.7 Recalling (4.47) and (4.51), it is easy to see that

$$
u_{0} \in D(\mathfrak{F}), f \in B V(0, T ; \mathscr{B}) \quad \Rightarrow \quad \Omega\left(\delta ; u_{0}, f\right) \leq \sqrt{\delta \rho\left(u_{0}, f\right)} .
$$

A standard density argument yields

$$
\lim _{\delta \downarrow 0} \Omega(\delta ; w, f)=0 \quad \forall w \in \overline{D(\mathfrak{F})}, f \in L^{1}(0, T ; \mathscr{B}) .
$$

The proof of Theorem 5 is based on the next three lemmas concerning extension, comparison, and stability of relaxed solution.

Lemma 6.8 (Extension) Let $\boldsymbol{u}:=(\tilde{u}, \bar{u})$ be a strong (weak) relaxed solution w.r.t. $u_{0}, f$ in $(0, T)$ and let $z \in D(\mathfrak{F}), \hat{z} \in \mathfrak{F}(z)$ be given. If we extend $\boldsymbol{u}, f, \xi$ for $t<0$ as

$$
\tilde{u}(t, w):=\tilde{u}(0, w), \quad \bar{u}(t):=z, \quad \xi(t) \equiv f(t):=\hat{z},
$$

then $\boldsymbol{u}$ is a strong (weak) relaxed solution w.r.t. $u_{0}, f$ in $(-\varepsilon, T)$ for all $\varepsilon \geq 0$.

Proof. It is a simple verification of $(6.3)$ or $(6.5)$ in $\mathscr{D}^{\prime}(-\varepsilon, T)$.

Lemma 6.9 (Comparison) Let us fix $\varepsilon>0$ and assume that

$$
\boldsymbol{u}:=(\tilde{u}, \bar{u}) \text { is a relaxed solution w.r.t. } u_{0}, f \text { in the interval }(-\varepsilon, T) \text {, }
$$

$\boldsymbol{v}:=(\tilde{v}, \bar{v})$ is a relaxed solution w.r.t. $v_{0}, g$ in the interval $(0, T)$,

and at least one of them is strong. Then we have

$$
\begin{aligned}
\int_{T-\varepsilon}^{T}(\tilde{v}(T, \bar{u}(s)) & \left.+\delta_{\boldsymbol{u}}(s)\right) d s \leq \int_{-\varepsilon}^{0}\left(\tilde{v}(0, \bar{u}(s))+\delta_{\boldsymbol{u}}(s)\right) d s \\
& +\int_{Q_{0, T}^{\varepsilon}}\|f(s)-g(t)\| d s d t+2\left(\Delta_{\boldsymbol{u}}+\Delta_{\boldsymbol{v}}\right)
\end{aligned}
$$

where $Q_{0, T}^{\varepsilon}$ denotes the strip (2.9) of Figure 1.

Remark 6.10 To see that this is indeed a comparison result, we apply it to Example 6.2 with $\boldsymbol{u}=(\|U-w\|, \bar{U})$ and $\boldsymbol{v}=(\|u-w\|, u), u$ being an integral solution of (CP) (see Remark 6.5). We thus get an estimate for the error

$$
\begin{aligned}
\int_{T-\varepsilon}^{T}\|u(T)-U(s)\| d s & \leq \int_{T-\varepsilon}^{T}(\|u(T)-\bar{U}(s)\|+\|\bar{U}(s)-U(s)\|) d s \\
& =\int_{T-\varepsilon}^{T}\left(\tilde{v}(T, \bar{u}(s))+\delta_{\boldsymbol{u}}(s)\right) d s
\end{aligned}
$$

Proof of Lemma 6.9. It is not restrictive to assume that $\boldsymbol{u}$ is a strong relaxed solution. Let $\mathbf{e}=\left(\mathrm{e}_{1}, \mathrm{e}_{2}\right), \mathbf{h}=\left(\mathrm{h}_{1}, \mathrm{~h}_{2}\right)$ be the auxiliary vector fields given by

$$
\begin{gathered}
\mathbf{e}(s, t):=(\tilde{u}(s, \bar{v}(t)), \tilde{v}(t, \bar{u}(s))) \\
\mathbf{h}(s, t):=\left([\xi(s)-f(s), \bar{u}(s)-\bar{v}(t)]_{+},[\xi(s)-g(t), \bar{v}(t)-\bar{u}(s)]_{-}\right) .
\end{gathered}
$$


Then (6.17) and (6.18) yield

$$
\begin{aligned}
& \frac{\partial}{\partial s} \mathrm{e}_{1}(s, t)+\mathrm{h}_{1}(s, t) \leq 0, \quad \text { in } \mathscr{D}^{\prime}(-\varepsilon, T) \quad \text { for a.e. } t \in(0, T), \\
& \frac{\partial}{\partial t} \mathrm{e}_{2}(s, t)+\mathrm{h}_{2}(s, t) \leq 0, \quad \text { in } \mathscr{D}^{\prime}(0, T) \quad \text { for a.e. } s \in(-\varepsilon, T),
\end{aligned}
$$

whence

$$
\operatorname{div} \mathbf{e}(s, t)+\mathrm{h}(s, t) \leq 0 \quad \text { in } \mathscr{D}^{\prime}((-\varepsilon, T) \times(0, T))
$$

with

$$
\begin{aligned}
\mathrm{h}(s, t) & :=\mathrm{h}_{1}(s, t)+\mathrm{h}_{2}(s, t) \\
& \geq-[f(s)-g(t), \bar{u}(s)-\bar{v}(t)]_{+} \geq-\|f(s)-g(t)\| .
\end{aligned}
$$

In order to prove (6.19), we simply have to apply (a slightly modified version of) the Divergence Theorem in the domain $Q_{0, T}^{\varepsilon}$ of Figure 1; see Lemma 6.15 at the end of this section for a rigorous proof. This gives, at least formally,

$$
\begin{aligned}
\int_{T-\varepsilon}^{T} \mathrm{e}_{2}(s, T) d s \leq \int_{-\varepsilon}^{0} \mathrm{e}_{2}(s, 0) d s \\
\quad+\int_{0}^{T}\left(\mathrm{e}_{2}(t, t)-\mathrm{e}_{1}(t, t)\right)+\left(\mathrm{e}_{1}(t-\varepsilon, t)-\mathrm{e}_{2}(t-\varepsilon, t)\right) d t \\
\quad+\int_{Q_{0, T}^{\varepsilon}}\|f(s)-g(t)\| d s d t
\end{aligned}
$$

Using the definitions of $\delta_{\boldsymbol{u}}(t)$ and $\delta_{\boldsymbol{v}}(t)$, we obtain

$$
\begin{aligned}
\mathrm{e}_{2}(t, t)-\mathrm{e}_{1}(t, t) & =\tilde{v}(t, \bar{u}(t))-\|\bar{u}(t)-\bar{v}(t)\| \\
& +\|\bar{u}(t)-\bar{v}(t)\|-\tilde{u}(t, \bar{v}(t)) \leq \delta_{\boldsymbol{v}}(t)+\delta_{\boldsymbol{u}}(t),
\end{aligned}
$$

and

$$
\begin{aligned}
\mathrm{e}_{1}(t-\varepsilon, t) & -\mathrm{e}_{2}(t-\varepsilon, t)=\tilde{u}(t-\varepsilon, \bar{v}(t))-\|\bar{u}(t-\varepsilon)-\bar{v}(t)\| \\
& +\|\bar{u}(t-\varepsilon)-\bar{v}(t)\|-\tilde{v}(t, \bar{u}(t-\varepsilon)) \leq \delta_{\boldsymbol{u}}(t-\varepsilon)+\delta_{\boldsymbol{v}}(t) .
\end{aligned}
$$

Upon adding $\int_{T-\varepsilon}^{T} \delta_{\boldsymbol{u}}(s) d s$ to the left-hand side of (6.27), and extracting the quantity $\int_{-\varepsilon}^{0} \delta_{\boldsymbol{u}}(s) d s$ from (6.28), we get the asserted estimate (6.19).

Corollary 1 Let us fix $\varepsilon>0$ and assume that

$$
\boldsymbol{u}:=(\tilde{u}, \bar{u}) \text { is a relaxed solution w.r.t. } u_{0}, f \text { in the interval }(0, T) \text {, }
$$

$\boldsymbol{v}:=(\tilde{v}, \bar{v})$ is a relaxed solution w.r.t. $v_{0}, g$ in the interval $(0, T)$,

and at least one of them is strong. Then we have

$$
\begin{aligned}
f_{T-\varepsilon}^{T}\left(\tilde{v}(T, \bar{u}(s))+\delta_{\boldsymbol{u}}(s)\right) d s & \leq\left\|u_{0}-v_{0}\right\|+\|f-g\|_{L^{1}(0, T ; \mathscr{B})} \\
& +2\left(\omega\left(\varepsilon / 4 ; u_{0}, f\right)+\frac{\Delta_{\boldsymbol{u}}+\Delta_{\boldsymbol{v}}}{\varepsilon}\right) .
\end{aligned}
$$


Proof. We fix $z \in D(\mathfrak{F}), \hat{z} \in \mathfrak{F}(z)$, extend $\tilde{u}, \bar{u}, f$ for $t<0$ as in Lemma 6.8, and apply Lemma 6.9. In view of (6.4) and (6.6), the first two terms on the right-hand side of (6.19) become

$$
\begin{gathered}
\int_{-\varepsilon}^{0} \tilde{v}(0, \bar{u}(s)) d s \leq \varepsilon\left\|v_{0}-z\right\| \leq \varepsilon\left\|v_{0}-u_{0}\right\|+\varepsilon\left\|u_{0}-z\right\|, \\
\int_{-\varepsilon}^{0} \delta_{\boldsymbol{u}}(s) d s \leq \varepsilon\left\|u_{0}-z\right\|,
\end{gathered}
$$

whereas the integral term in $Q_{0, T}^{\varepsilon}$ can be bounded by

$$
\begin{aligned}
\int_{Q_{0, T}^{\varepsilon}}\|f(s)-g(t)\| d s d t & \leq \int_{Q_{0, T}^{\varepsilon}}(\|f(t)-g(t)\|+\|f(s)-f(t)\|) d s d t \\
& =\varepsilon \int_{0}^{T}\|f(t)-g(t)\| d t+\int_{Q_{0, T}^{\varepsilon}}\|f(s)-f(t)\| d s d t .
\end{aligned}
$$

The last integral can be estimated as follows for every $\psi \in B V(0, T ; \mathscr{B})$ :

$$
\int_{Q_{0, T}^{\varepsilon}}\|f(s)-f(t)\| d s d t \leq 2 \varepsilon\|f-\psi\|_{L^{1}(0, T ; \mathscr{B})}+\frac{\varepsilon^{2}}{2}(\|\psi(0)-\hat{z}\|+\operatorname{Var} \psi) .
$$

In fact, setting $\psi(t) \equiv \hat{z}$ for $t<0$, we have

$$
\begin{aligned}
\int_{Q_{0, T}^{\varepsilon}} & \|f(s)-f(t)\| d s d t \\
\quad \leq & \int_{Q_{0, T}^{\varepsilon}}(\|f(s)-\psi(s)\|+\|\psi(s)-\psi(t)\|+\|\psi(t)-f(t)\|) d s d t \\
& \leq 2 \varepsilon\|f-\psi\|_{L^{1}(0, T ; \mathscr{B})}+\int_{0}^{T}\left(\int_{0}^{\varepsilon}\|\psi(t)-\psi(t-h)\| d h\right) d t,
\end{aligned}
$$

and, invoking (4.41),

$$
\begin{aligned}
\int_{0}^{T} & \left(\int_{0}^{\varepsilon}\|\psi(t)-\psi(t-h)\| d h\right) d t \\
& \leq \int_{0}^{\varepsilon}\left(\int_{0}^{h \wedge T}\|\psi(t)-\hat{z}\| d t+\int_{h \wedge T}^{T}\|\psi(t)-\psi(t-h)\| d t\right) d h \\
& \leq \int_{0}^{\varepsilon}(h \wedge T)(\|\hat{z}-\psi(0)\|+\operatorname{Var} \psi) d h \leq \frac{\varepsilon^{2}}{2}(\|\hat{z}-\psi(0)\|+\operatorname{Var} \psi) .
\end{aligned}
$$

Hereafter, we use the notation

$$
a \wedge b:=\min (a, b), \quad a \vee b:=\max (a, b) .
$$

Substituting these estimates into (6.19) and taking the infimum w.r.t. $z, \hat{z}$ the definition (4.49) of $\omega$ yields (6.31).

Now we establish a sort of stability estimate for strong relaxed solutions. 
Lemma 6.11 (Stability) Let $\varepsilon>0$ be fixed and let $\boldsymbol{u}:=(\tilde{u}, \bar{u})$ be a strong relaxed solution w.r.t. $u_{0}, f$ in $(0, T)$. We have

$$
f_{T-\varepsilon}^{T}\left(\tilde{u}(T, \bar{u}(s))+\delta_{\boldsymbol{u}}(s)\right) d s \leq 2\left(\omega\left(\varepsilon / 4 ; u_{0}, f\right)+\frac{\Delta_{\boldsymbol{u}}}{\varepsilon}\right) .
$$

Proof. We apply the same extension argument of the previous corollary and the same reasoning of Lemma 6.9 to the couples $\boldsymbol{u}=\boldsymbol{v}=(\tilde{u}, \bar{u})$. We observe though that in this case the vector field e defined by (6.21) satisfies $\mathrm{e}_{1}(t, t) \equiv$ $\mathrm{e}_{2}(t, t)$. Therefore, (6.27) and (6.28) become

$$
\begin{aligned}
\int_{T-\varepsilon}^{T}\left(\tilde{u}(T, \bar{u}(s))+\delta_{\boldsymbol{u}}(s)\right) d s & \leq \int_{-\varepsilon}^{0} \tilde{u}(0, \bar{u}(s)) d s \\
& +\int_{-\varepsilon}^{0}(\tilde{u}(s, \bar{u}(s+\varepsilon))-\|\bar{u}(s)-\bar{u}(s+\varepsilon)\|) d s \\
& +\int_{Q_{0, T}^{\varepsilon}}\|f(s)-f(t)\| d s d t+2 \Delta_{\boldsymbol{u}} .
\end{aligned}
$$

In view of $(6.16)$, we readily have $\tilde{u}(0, \bar{u}(s)) \leq\left\|u_{0}-z\right\|$ and $\tilde{u}(s, w)-\|\bar{u}(s)-w\| \leq$ $\left\|u_{0}-z\right\|$ for $-\varepsilon<s<0$. Finally, arguing as in Corollary 1, we obtain (6.35).

Remark 6.12 To verify that Lemma 6.11 gives indeed an estimate of the "time regularity" of $\boldsymbol{u}$, we apply it to Example 6.2 with $\boldsymbol{u}=(\|U-w\|, \bar{U})$. We obtain

$$
\begin{aligned}
f_{T-\varepsilon}^{T} \| U(T) & -U(s) \| d s \leq f_{T-\varepsilon}^{T}\left(\tilde{u}(T, \bar{u}(s))+\delta_{\boldsymbol{u}}(s)\right) d s \\
& \leq 2\left(\omega\left(\varepsilon / 4 ; U_{0}, \bar{F}\right)+\frac{\Delta_{\boldsymbol{u}}}{\varepsilon}\right)=\frac{\varepsilon}{2}\left(\left\|F_{1}-\mathfrak{F}\left(U_{0}\right)\right\|+\operatorname{Var} \bar{F}\right)+\frac{2 \Delta_{\boldsymbol{u}}}{\varepsilon} .
\end{aligned}
$$

We then see that this extends $(2.13)$ to nonzero forcing $\bar{F}$, and realize the presence of the additional term $\frac{2 \Delta \boldsymbol{u}}{\varepsilon}$. This result is not sharp for Example 6.2.

Remark 6.13 If $u$ is a weak integral solution of (CP) and $\boldsymbol{u}=(\|u-w\|, u)$, as in Remark 6.5, then (4.52) gives (6.35) directly.

Proof of Theorem 5. We add (6.31) and (6.35), and observe that

$$
\int_{T-\varepsilon}^{T}(\tilde{v}(T, \bar{u}(s))+\tilde{u}(T, \bar{u}(s))) d s \geq \varepsilon \mathfrak{D}(T ; \boldsymbol{u}, \boldsymbol{v}),
$$

to end up with

$$
\mathfrak{D}(T ; \boldsymbol{u}, \boldsymbol{v}) \leq\left\|u_{0}-v_{0}\right\|+\|f-g\|_{L^{1}(0, T ; \mathscr{B})}+4 \omega\left(\varepsilon / 4 ; u_{0}, f\right)+\frac{4 \Delta_{\boldsymbol{u}}+2 \Delta_{\boldsymbol{v}}}{\varepsilon} .
$$

Taking the infimum w.r.t. $\varepsilon>0$, we get the asserted estimate (6.12).

Remark 6.14 Since the comparison Lemma 6.9 and its Corollary 1 make no distinction between strong and weak solution, so that the modulus of regularity $\omega$ of either $\boldsymbol{u}$ or $\boldsymbol{v}$ could be used, we may wonder about the assumption that $\boldsymbol{u}$ is a strong relaxed solution. This enters in the main estimate (6.12) of Theorem 5 via Lemma 6.11, and is consistent with step $\mathrm{V}$ of $\S 2$. The following result reverts this situation provided $u$ is a weak integral solution. 
Corollary 2 Let $u$ be an integral solution of $(\mathrm{CP})$ and $\tilde{u}(t, w):=\|u(t)-w\|$, $\bar{u}(t):=u(t)$ be as in (6.11) so that

$\boldsymbol{u}:=(\tilde{u}, \bar{u})$ is a weak relaxed solution w.r.t. $u_{0}, f$ in the interval $(0, T)$

with discrepancy $\Delta_{\boldsymbol{u}}=0$. Let

$\boldsymbol{v}:=(\tilde{v}, \bar{v})$ be a strong relaxed solution w.r.t. $v_{0}, g$ in the interval $(0, T)$.

Then, the deviation $\mathfrak{D}(T ; \boldsymbol{u}, \boldsymbol{v})$ of $\boldsymbol{u}$ and $\boldsymbol{v}$ at time $T$ satisfies

$$
\mathfrak{D}(T ; \boldsymbol{u}, \boldsymbol{v}) \leq\left\|u_{0}-v_{0}\right\|+\|f-g\|_{L^{1}(0, T ; \mathscr{B})}+4 \Omega\left(\frac{1}{2} \Delta_{\boldsymbol{v}} ; u_{0}, f\right) .
$$

Proof. Argue as in Theorem 5 with Remark 6.13 instead of Lemma 6.11.

The following lemma establishes (6.27) in a weak context where (6.23) and (6.24) are only known to hold in the sense of distributions. This is not a difficult task because of the simple geometry of $Q_{0, T}^{\varepsilon}$ defined in (2.9).

Lemma 6.15 (A Weak Divergence Theorem) Let $\mathbf{e}=\left(\mathrm{e}_{1}, \mathrm{e}_{2}\right)$ and $\mathbf{h}=$ $\left(\mathrm{h}_{1}, \mathrm{~h}_{2}\right)$ be integrable vector fields in $(-\varepsilon, T) \times(0, T)$ with

$$
\begin{aligned}
& s \mapsto \mathrm{e}_{1}(s, t) \in C^{0}([-\varepsilon, T]) \quad \text { for a.e. } t \in(0, T), \\
& t \mapsto \mathrm{e}_{2}(s, t) \in C^{0}([0, T]) \quad \text { for a.e. } s \in(-\varepsilon, T) .
\end{aligned}
$$

If they satisfy (6.23) and (6.24), then (6.27) holds.

ProOF. Let us start from (6.23) in the equivalent integral form (4.37) with $\alpha:=t-\varepsilon, \beta:=t$ for a.e. $t \in[0, T]$ :

$$
\mathrm{e}_{1}(t, t)-\mathrm{e}_{1}(t-\varepsilon, t)+\int_{t-\varepsilon}^{t} \mathrm{~h}_{1}(s, t) d s \leq 0 .
$$

If we integrate this inequality from $t=0$ to $t=T$, we get

$$
\int_{0}^{T}\left(\mathrm{e}_{1}(t, t)-\mathrm{e}_{1}(t-\varepsilon, t)\right) d t+\int_{Q_{0, T}^{\varepsilon}} \mathrm{h}_{1}(s, t) d s d t \leq 0 .
$$

We now write the integral form of (6.24) between $\alpha=s \vee 0$ and $\beta=(s+\varepsilon) \wedge T$, and thereby obtain as before for a.e. $s \in(-\varepsilon, T)$

$$
\mathrm{e}_{2}(s,(s+\varepsilon) \wedge T)-\mathrm{e}_{2}(s, s \vee 0)+\int_{s \vee 0}^{(s+\varepsilon) \wedge T} \mathrm{~h}_{2}(s, t) d t \leq 0 .
$$

Finally we perform another integration w.r.t. $s$ from $-\varepsilon$ to $T$

$$
\int_{-\varepsilon}^{T}\left(\mathrm{e}_{2}(s,(s+\varepsilon) \wedge T)-\mathrm{e}_{2}(s, s \vee 0)\right) d s+\int_{Q_{0, T}^{\varepsilon}} \mathrm{h}_{2}(s, t) d s d t \leq 0 .
$$

We observe that the first integral of this inequality can be rewritten as

$$
\begin{aligned}
& \int_{-\varepsilon}^{T}\left(\mathrm{e}_{2}(s,(s+\varepsilon) \wedge T)-\mathrm{e}_{2}(s, s \vee 0)\right) d s= \\
& \int_{0}^{T} \mathrm{e}_{2}(t-\varepsilon, t) d t+\int_{T-\varepsilon}^{T} \mathrm{e}_{2}(s, T) d s-\int_{-\varepsilon}^{0} \mathrm{e}_{2}(s, 0) d s-\int_{0}^{T} \mathrm{e}_{2}(t, t) d t .
\end{aligned}
$$

Substituting (6.41) into (6.40), and adding (6.39), we get (6.27). 


\section{A Posteriori and A Priori Error Estimates}

In this section we derive error estimates, both a posteriori and a priori, for the discrete solutions (1.4), (1.5) of the implicit Euler scheme $\left(\mathrm{IS}_{1}\right)$ and the explicit Euler method $\left(\mathrm{ES}_{1}\right)$, as well as the Yosida regularization of $\S 5.3$.

\subsection{Implicit Euler Method}

Theorem 1 for $\left(\mathrm{IS}_{1}\right)$ could be easily deduced as a direct application of Theorem 5 , but with a stability constant $2 \sqrt{2}$ instead of 2 (see Theorem 1 ). To show a constant 2 , we present first a slight refinement of the stability estimate (6.35).

Lemma 7.1 (Strong Stability) Let $\left\{U_{n}\right\}_{n=1}^{N},\left\{V_{n}\right\}_{n=0}^{N}$ be the discrete solutions of the implicit Euler scheme (IS $\mathrm{IS}_{1,2,3}$ ) with respect to the initial values $U_{0}, V_{0}$ and the discrete source terms $\left\{F_{n}\right\}_{n=1}^{N},\left\{G_{n}\right\}_{n=1}^{N}$. Then

$$
\sup _{n=1, \ldots, N}\left\|U_{n}-V_{n}\right\| \leq\left\|U_{0}-V_{0}\right\|+\|\bar{F}-\bar{G}\|_{L^{1}(0, T ; \mathscr{B})},
$$

and

$$
\sup _{n=1, \ldots, N} \frac{1}{\tau_{n}}\left\|U_{n}-U_{n-1}\right\| \leq\left\|F_{1}-\mathfrak{F}\left(U_{0}\right)\right\|+\operatorname{Var} \bar{F}=\rho\left(U_{0}, F\right) .
$$

In addition,

$$
\begin{aligned}
|t-s| \leq \varepsilon \Rightarrow\|U(t)-U(s)\| & \leq 2 \omega\left(\varepsilon / 2 ; U_{0}, \bar{F}\right) \\
& \leq \varepsilon\left(\left\|F_{1}-\mathfrak{F}\left(U_{0}\right)\right\|+\operatorname{Var} \bar{F}\right) .
\end{aligned}
$$

Proof. We first note that (7.1) follows directly from the accretiveness $\left(\mathrm{IS}_{3}\right)$ of $\mathfrak{F}$, which gives

$$
\left\|U_{n}-V_{n}\right\| \leq\left\|U_{n-1}-V_{n-1}\right\|+\tau_{n}\left\|F_{n}-G_{n}\right\| \quad \forall n=1, \ldots, N .
$$

In order to show (7.2), let us set

$$
U_{-1}:=U_{0}, \quad F_{0}:=\mathfrak{F}\left(U_{0}\right), \quad \tau_{0}>0,
$$

so that $\left(\mathrm{IS}_{1}\right)$ holds also for $n=0$. Taking the difference of two consecutive discrete equations $\left(\mathrm{IS}_{1}\right)$, and using $(4.6 \mathrm{~b})$, we get

$$
\begin{aligned}
{\left[\tau_{n}^{-1}\left(U_{n}-U_{n-1}\right)\right.} & \left.-\tau_{n-1}^{-1}\left(U_{n-1}-U_{n-2}\right)-\left(F_{n}-F_{n-1}\right), U_{n}-U_{n-1}\right]_{-} \\
& +\left[\mathfrak{F}\left(U_{n}\right)-\mathfrak{F}\left(U_{n-1}\right), U_{n}-U_{n-1}\right]_{+} \leq 0 .
\end{aligned}
$$

Hence, by the accretiveness $\left(\mathrm{IS}_{3}\right)$ of $\mathfrak{F}$ and $(4.2 \mathrm{~b})$,

$$
\frac{1}{\tau_{n}}\left\|U_{n}-U_{n-1}\right\|-\frac{1}{\tau_{n-1}}\left\|U_{n-1}-U_{n-2}\right\|-\left\|F_{n}-F_{n-1}\right\| \leq 0 \quad \forall 1 \leq n \leq N .
$$

Summing over $n$, for $n=1$ to $n=m \leq N$, we arrive at (7.2).

Since $V(t)$ is continuous and piecewise linear, and

$$
V^{\prime}(t)=\frac{V_{n}-V_{n-1}}{\tau_{n}} \quad \forall t \in\left(t_{n-1}, t_{n}\right),
$$


then (7.2) yields

$$
|t-s| \leq \varepsilon \quad \Rightarrow \quad\|V(t)-V(s)\| \leq \varepsilon\left(\left\|G_{1}-\mathfrak{F}\left(V_{0}\right)\right\|+\operatorname{Var} \bar{G}\right) .
$$

Therefore

$$
\begin{aligned}
\|U(t)-U(s)\| & \leq 2 \sup _{r \in[0, T]}\|U(r)-V(r)\|+\|V(t)-V(s)\| \\
& \leq 2\left(\left\|U_{0}-V_{0}\right\|+\|\bar{F}-\bar{G}\|_{L^{1}(0, T ; \mathscr{B})}\right)+\varepsilon \rho\left(V_{0}, \bar{G}\right) .
\end{aligned}
$$

Taking the infimum with respecto to $V_{0}$ and $\bar{G}$, (4.56) and (4.51) imply (7.3), and conclude the proof.

Corollary 3 (Error Estimates) Let $u$ be the integral solution of (CP). Let $\left\{U_{n}\right\}_{n=0}^{N}$ be the solution of $\left(I S_{1,2,3}\right)$ and let $U_{0} \in D(\mathfrak{F})$. The following a posteriori error estimate is valid

$$
\|u(T)-U(T)\| \leq\left\|u_{0}-U_{0}\right\|+\|f-\bar{F}\|_{L^{1}(0, T ; \mathscr{B})}+\mathscr{E}_{\tau}\left(U_{0}, \bar{F}\right),
$$

where the estimator $\mathscr{E}_{\tau}\left(U_{0}, \bar{F}\right)$ is given by

$$
\begin{aligned}
\mathscr{E}_{\tau}\left(U_{0}, \bar{F}\right) & =4 \Omega\left(\frac{1}{4} \sum_{n=1}^{N} \tau_{n}\left\|U_{n}-U_{n-1}\right\| ; U_{0}, \bar{F}\right) \\
& \leq 2\left(\left\|F_{1}-\mathfrak{F}\left(U_{0}\right)\right\|+\operatorname{Var} F\right)^{1 / 2}\left(\sum_{n=1}^{N} \tau_{n}\left\|U_{n}-U_{n-1}\right\|\right)^{1 / 2} .
\end{aligned}
$$

Moreover, the following a priori estimate holds

$$
\sum_{n=1}^{N} \tau_{n}\left\|U_{n}-U_{n-1}\right\| \leq T\left(\left\|F_{1}-\mathfrak{F}\left(U_{0}\right)\right\|+\operatorname{Var}(\bar{F})\right) \tau .
$$

Proof. In view of Theorem 2 and Example 6.2, the pair $\boldsymbol{u}:=(\|U-w\|, \bar{U})$ is a strong solution w.r.t. $U_{0}, \bar{F}$ of $(\mathrm{CP})$ with $\Delta_{\boldsymbol{u}}=\frac{1}{2} \sum_{n=1}^{N} \tau_{n}\left\|U_{n}-U_{n-1}\right\|$. Moreover, (7.3) implies

$$
f_{T-\varepsilon}^{T}\|U(T)-U(s)\| \leq \frac{\varepsilon}{2}\left(\left\|F_{1}-\mathfrak{F}\left(U_{0}\right)\right\|+\operatorname{Var} \bar{F}\right) .
$$

Since the pair $\boldsymbol{v}=(\|u-w\|, u)$ is a relaxed solution w.r.t. $u_{0}, f$ of $(\mathrm{CP})$ with $\Delta_{\boldsymbol{v}}=0$, applying Corollary 1 , we get

$$
\begin{aligned}
f_{T-\varepsilon}^{T} \| u(T) & -U(s) \| d s \leq f_{T-\varepsilon}^{T}\left(\|u(T)-\bar{U}(s)\|+\delta_{\boldsymbol{u}}(s)\right) d s \\
& \leq\left\|u_{0}-U_{0}\right\|+\|f-F\|_{L^{1}(0, T ; \mathscr{B})}+2 \omega\left(\varepsilon / 4 ; U_{0}, \bar{F}\right)+\frac{2}{\varepsilon} \Delta_{\boldsymbol{u}} .
\end{aligned}
$$

Adding this estimate with (7.9), we readily obtain

$$
\begin{aligned}
\|U(T)-u(T)\| & \leq\left\|u_{0}-U_{0}\right\|+\|f-F\|_{L^{1}(0, T ; \mathscr{B})} \\
& +4 \omega\left(\varepsilon / 4 ; U_{0}, \bar{F}\right)+\frac{1}{\varepsilon} \sum_{n=1}^{N} \tau_{n}\left\|U_{n}-U_{n-1}\right\| .
\end{aligned}
$$

Finally, (7.7) follows upon taking the infimum w.r.t. $\varepsilon>0$ and using (7.3). On the other hand, (7.8) is a direct consequence of (7.2). 
Remark 7.2 If we were to use Remark 6.12, instead of Lemma 7.1, then (7.9) would have the additional summand $\frac{2}{\varepsilon} \Delta_{\boldsymbol{u}}$. Consequently, the last term in $(7.10)$ would have a factor $\frac{2}{\varepsilon}$ which, after optimization in $\varepsilon$, would lead to the worse stability constant $2 \sqrt{2}$ mentioned at the beginning of $\S 7.1$.

\subsection{Explicit Euler Method}

We now consider the solution of the explicit Euler scheme $\left(\mathrm{ES}_{1}\right)$. From Example 6.3 , we see that the couple $\boldsymbol{u}:=(\tilde{U}, \bar{U})$, with $\tilde{U}$ defined in $(5.4)$, is a weak relaxed solution w.r.t. $U_{0}, \bar{F}$ of $(\mathrm{CP})$. However, a direct application of Theorem 5 would give an a priori estimate in terms of $u_{0}, f$. To get around, we need a stability estimate for explicit discrete solutions similar to that of Lemma 7.1.

Lemma 7.3 (Strong Stability) Let $\left\{U_{n}\right\}_{n=1}^{N},\left\{V_{n}\right\}_{n=0}^{N}$ be the discrete solutions of the Explicit Euler scheme $\left(\mathrm{ES}_{1,2,3}\right)$ with respect to the initial values $U_{0}, V_{0}$ and discrete source terms $\left\{F_{n}\right\}_{n=1}^{N},\left\{G_{n}\right\}_{n=1}^{N}$. Then

$$
\sup _{n=1, \ldots, N}\left\|U_{n}-V_{n}\right\| \leq\left\|U_{0}-V_{0}\right\|+\|\bar{F}-\bar{G}\|_{L^{1}(0, T ; \mathscr{B})}
$$

and

$$
\sup _{n=1, \ldots, N} \frac{\left\|U_{n}-U_{n-1}\right\|}{\tau_{n}} \leq\left\|F_{1}-\mathfrak{F}\left(U_{0}\right)\right\|+\operatorname{Var} \bar{F}
$$

In addition,

$$
\begin{aligned}
|t-s| \leq \varepsilon \quad \Rightarrow \quad\|U(t)-U(s)\| & \leq 2 \omega\left(\varepsilon / 2 ; U_{0}, \bar{F}\right) \\
& \leq \varepsilon\left(\left\|F_{1}-\mathfrak{F}\left(U_{0}\right)\right\|+\operatorname{Var} \bar{F}\right) .
\end{aligned}
$$

Proof. We first observe that (7.11) follows directly from the explicit contractivity $\left(\mathrm{ES}_{3}\right)$ of $\mathfrak{F}$, or equivalently (4.19). For $n=1, \ldots, N$ we set

$$
W_{n}:=U_{n-1}+\tau_{n-1} F_{n}-\tau_{n-1} \mathfrak{F}\left(U_{n-1}\right),
$$

so that

$$
\frac{U_{n}-U_{n-1}}{\tau_{n}}=\frac{W_{n}-U_{n-1}}{\tau_{n-1}} .
$$

Using again (4.19) yields

$$
\left\|W_{n}-U_{n-1}-\tau_{n-1}\left(F_{n}-F_{n-1}\right)\right\| \leq\left\|U_{n-1}-U_{n-2}\right\|
$$

whence

$$
\frac{\left\|U_{n}-U_{n-1}\right\|}{\tau_{n}} \leq \frac{\left\|W_{n}-U_{n-1}\right\|}{\tau_{n-1}} \leq \frac{\left\|U_{n-1}-U_{n-2}\right\|}{\tau_{n-1}}+\left\|F_{n}-F_{n-1}\right\| .
$$

In order to deduce $(7.12)$, we set $U_{-1}:=U_{0}, F_{0}:=\mathfrak{F}\left(U_{0}\right)$, and observe that in this way $\left(\mathrm{ES}_{1}\right)$ holds for $n=0$ as well. We next sum up (7.14), from $n=1$ to $n=m \leq N$, to obtain

$$
\frac{\left\|U_{m}-U_{m-1}\right\|}{\tau_{m}} \leq\left\|F_{1}-\mathfrak{F}\left(U_{0}\right)\right\|+\sum_{n=2}^{m}\left\|F_{n}-F_{n-1}\right\| \quad \forall m=1, \ldots N .
$$

This gives (7.12). We finally proceed as in Lemma 7.1 to prove (7.13). 
Corollary 4 (Error Estimates) Let $\left(E S_{2,3}\right)$ be valid so that $\left\{U_{n}\right\}_{n=0}^{N}$, solution of the explicit scheme $\left(\mathrm{ES}_{1}\right)$, be well defined starting from $U_{0} \in \mathscr{B}$. Let $U, \bar{U}$ be defined as in (1.4), (1.5) and $u$ be the (strong) solution of (CP). Then

$$
\|u(T)-U(T)\| \leq\left\|u_{0}-U_{0}\right\|+\|f-\bar{F}\|_{L^{1}(0, T ; \mathscr{B})}+\mathscr{E}_{\tau}\left(U_{0}, \bar{F}\right),
$$

where the a posteriori error estimator $\mathscr{E}_{\tau}\left(U_{0}, \bar{F}\right)$ satisfies $(7.7)$ and (7.8).

Proof. According to Theorem 3 and Example $6.3, \boldsymbol{u}=(\tilde{U}, \bar{U})$ is a weak relaxed solution w.r.t. $U_{0}, F$ in $(0, T)$, with discrepancy $\Delta_{\boldsymbol{u}}=\frac{1}{2} \sum_{n=1}^{N} \tau_{n} \| U_{n}-$ $U_{n-1} \|$. On the other hand, the pair $\boldsymbol{v}=(\|u-w\|, u)$ is a strong relaxed solution with $\Delta_{\boldsymbol{v}}=0$. Therefore, applying Corollary 1 , in conjunction with Lemma 7.3, and arguing as in Corollary 3 leads to (7.15).

\subsection{Yosida Regularization}

We conclude this paper with an error estimate for the Yosida regularizion of $\S 4.1(d)$. As in Definition 4.10 we set

$$
\omega(\varepsilon ; f):=\inf \left\{\|f-\psi\|_{L^{1}(0, T ; \mathscr{B})}+\varepsilon\|\psi\|_{B V(0, T ; \mathscr{B})}: \psi \in B V(0, T ; \mathscr{B})\right\},
$$

which obviously satisfies

$$
\omega(\varepsilon ; f) \leq \varepsilon\|f\|_{B V(0, T ; \mathscr{B})} \quad \forall f \in B V(0, T ; \mathscr{B}) .
$$

Corollary 5 (Error Estimates) Let $u_{\tau}$ be the (strong) solution of (5.8) and let $u$ be the integral solution of $(\mathrm{CP})$. Then

$$
\begin{gathered}
\sup _{t \in[0, T]}\left\|u(t)-u_{\tau}(t)\right\| \leq 3 \omega\left(\frac{2}{3} \tau ; f\right)+4 \Omega\left(\frac{1}{2} \tau \operatorname{Var} u_{\tau}+\tau \omega\left(\frac{1}{2} \tau ; f\right) ; u_{0}, f\right) \\
\quad \leq 2 \tau\|f\|_{B V(0, T ; \mathscr{B})}+2 \sqrt{2}\left(\left(\tau \operatorname{Var} u_{\tau}+\tau^{2}\|f\|_{B V(0, T ; \mathscr{B})}\right) \rho\left(u_{0}, f\right)\right)^{\frac{1}{2}} .
\end{gathered}
$$

In particular, when $f=0$, we have

$$
\sup _{t \in[0, T]}\left\|u(t)-u_{\tau}(t)\right\| \leq 4 \Omega\left(\frac{T}{2} \tau\left\|\mathfrak{F}_{\tau}\left(u_{0}\right)\right\| ; u_{0}, 0\right) \leq 2 \sqrt{2 \tau T}\left\|\mathfrak{F}\left(u_{0}\right)\right\| .
$$

Proof. In light of Theorem 4 and Example 6.4, the couple $\boldsymbol{v}=\left(\left\|v_{\tau}-w\right\|, \bar{v}_{\tau}\right)$, defined in (5.10) with $v_{\tau}=u_{\tau}-\tau f_{\tau}$ and $\bar{v}_{\tau}=\mathfrak{J}_{\tau}\left(u_{\tau}\right)$, is a strong relaxed solution of (CP) w.r.t $u_{0}, f_{\tau}$ with discrepancy $\Delta_{\boldsymbol{v}}=\tau \operatorname{Var} v_{\tau}$. Since $\boldsymbol{u}=(\|u-w\|, u)$ is a weak relaxed solution of (CP) w.r.t $u_{0}, f$ with $\Delta_{\boldsymbol{u}}=0$ and

$$
\mathfrak{D}(T ; \boldsymbol{u}, \boldsymbol{v}) \geq\left\|u(T)-u_{\tau}(T)-\tau f_{\tau}(T)\right\| \geq\left\|u(T)-u_{\tau}(T)\right\|-\tau\left\|f_{\tau}(T)\right\|,
$$

we obtain from Corollary 2

$$
\begin{aligned}
\left\|u(T)-u_{\tau}(T)\right\| & \leq \tau\left\|f_{\tau}(T)\right\|+\mathfrak{D}(T ; \boldsymbol{u}, \boldsymbol{v}) \\
& \leq \tau\left\|f_{\tau}(T)\right\|+\left\|f-f_{\tau}\right\|_{L^{1}(0, T ; \mathscr{B})}+4 \Omega\left(\frac{\tau}{2} \operatorname{Var} v_{\tau} ; u_{0}, f\right) .
\end{aligned}
$$

Now we observe that

$$
\tau\left\|f_{\tau}(T)\right\| \leq\|f\|_{L^{1}(0, T ; \mathscr{B})}, \quad\left\|f-f_{\tau}\right\|_{L^{1}(0, T ; \mathscr{B})} \leq 2\|f\|_{L^{1}(0, T ; \mathscr{B})},
$$


and, invoking (5.9) and $f \in B V(0, T ; \mathscr{B})$,

$$
\tau\left\|f_{\tau}(T)\right\| \leq \tau \operatorname{Var} f_{\tau}=\left\|f-f_{\tau}\right\|_{L^{1}(0, T ; \mathscr{B})} \leq \tau(\operatorname{Var} f+\|f(0)\|) .
$$

Since the map $f \mapsto f_{\tau}$ defined by (5.9) is linear, we easily get

$$
\begin{aligned}
& \tau\left\|f_{\tau}(T)\right\|+\left\|f-f_{\tau}\right\|_{L^{1}(0, T ; \mathscr{B})} \leq \\
& \inf _{\psi \in B V(0, T ; \mathscr{B})}\left\{3\|f-\psi\|_{L^{1}(0, T ; \mathscr{B})}+2 \tau(\operatorname{Var} \psi+\|\psi(0)\|)\right\}=3 \omega\left(\frac{2}{3} \tau ; f\right) .
\end{aligned}
$$

Analogously

$$
\tau \operatorname{Var} f_{\tau}=\left\|f-f_{\tau}\right\|_{L^{1}(0, T ; \mathscr{B})} \leq 2 \omega\left(\frac{1}{2} \tau ; f\right),
$$

and in particular

$$
\frac{\tau}{2} \operatorname{Var} v_{\tau} \leq \frac{\tau}{2}\left(\operatorname{Var} u_{\tau}+\tau \operatorname{Var} f_{\tau}\right) \leq \frac{1}{2} \tau \operatorname{Var} u_{\tau}+\tau \omega\left(\frac{1}{2} \tau ; f\right) .
$$

We thus obtain (7.18); taking into account (6.14) and (7.17), we also get (7.19). Finally, (7.20) follows by observing that $\rho\left(u_{0}, 0\right)=\left\|\mathfrak{F}\left(u_{0}\right)\right\|$ and

$$
\operatorname{Var} u_{\tau} \leq T \sup _{t \in[0, T]}\left\|\mathfrak{F}_{\tau}\left(u_{\tau}\right)\right\| \leq T\left\|\mathfrak{F}_{\tau}\left(u_{0}\right)\right\| \leq T\left\|\mathfrak{F}\left(u_{0}\right)\right\|,
$$

where the last inequality results from (4.17). This concludes the proof.

\section{References}

[1] L. Ambrosio, N. Gigli, And G. SAvaré, Gradient flows in metric spaces and in spaces of probability measures, ETH Lecture notes, Birkhäuser, 2004. To appear.

[2] V. Barbu, Nonlinear Semigroups and Differential Equations in Banach Spaces, Editura Academiei Republicii Socialiste România, Bucharest, 1976. Translated from the Romanian.

[3] G. Barles And P. E. Souganidis, Convergence of approximation schemes for fully nonlinear second order equations, Asymptotic Anal., 4 (1991), pp. 271-283.

[4] P. BÉnILAn, Solutions intégrales d'équations d'évolution dans un espace de Banach, C. R. Acad. Sci. Paris Sér. A-B, 274 (1972), pp. A47-A50.

[5] J. Bergh AND J. LÖFström, Interpolation Spaces. An introduction, Springer-Verlag, Berlin, 1976. Grundlehren der Mathematischen Wissenschaften, No. 223.

[6] D. BrÉzIs, Classes d'interpolation associées à un opérateur monotone, C. R. Acad. Sci. Paris Sér. A-B, 276 (1973), pp. A1553-A1556.

[7] H. BRÉZIs, Opérateurs Maximaux Monotones et Sémi-groupes de Contractions dans les Espaces de Hilbert, North Holland, Amsterdam, 1973.

[8] H. Brezis, Analyse Fonctionnelle - Théorie et Applications, Masson, Paris, 1983. 
[9] H. Brézis And W. A. Strauss, Semi-linear second-order elliptic equations in $L^{1}$, J. Math. Soc. Japan, 25 (1973), pp. 565-590.

[10] B. Cockburn and G. Gripenberg, Continuous dependence of the nonlinearities of solutions of degenerate parabolic equations, J. Differential Equations 151, 151 (1999), pp. 231-251.

[11] M. G. Crandall, The semigroup approach to first order quasilinear equations in several space variables, Israel J. Math., 12 (1972), pp. 108-132.

[12] M. G. Crandall and L. C. Evans, On the relation of the operator $\partial / \partial s+\partial / \partial \tau$ to evolution governed by accretive operators, Israel J. Math., 21 (1975), pp. 261-278.

[13] M. G. Crandall, L. C. Evans, and P.-L. Lions, Some properties of viscosity solutions of Hamilton-Jacobi equations, Trans. Amer. Math. Soc., 282 (1984), pp. 487-502.

[14] M. G. Crandall, H. IshiI, and P.-L. Lions, User's guide to viscosity solutions of second order partial differential equations, Bull. Amer. Math. Soc. (N.S.), 27 (1992), pp. 1-67.

[15] M. G. Crandall and T. M. Liggett, Generation of semi-groups of nonlinear transformations on general Banach spaces, Amer. J. Math., 93 (1971), pp. 265-298.

[16] M. G. Crandall and P.-L. Lions, Viscosity solutions of HamiltonJacobi equations, Trans. Amer. Math. Soc., 277 (1983), pp. 1-42.

[17] M. G. Crandall And L. TARTaR, Some relations between nonexpansive and order preserving mappings., Proc. Am. Math. Soc., 78 (1980).

[18] G. Da Prato, Applications croissantes et équations d'évolutions dans les espaces de Banach, vol. II of Institutiones Mathematicae, Academic Press, London, New York, 1976.

[19] R. Eymard, T. Gallouet, and R. Herbin, Finite volume methods, Handbook of Numerical Analysis, VII (2000), pp. 713-1020.

[20] Y. Kobayashi, Difference approximation of Cauchy problems for quasidissipative operators and generation of nonlinear semigroups, J. Math. Soc. Japan, 27 (1975), pp. 640-665.

[21] D. KRÖNER, Numerical Schemes for Conservation Laws, John Wiley \& Sons Ltd., Chichester, 1997.

[22] S. N. KRUžKov, First order quasilinear equations with several independent variables., Mat. Sb. (N.S.), 81 (123) (1970), pp. 228-255.

[23] N. N. Kuznetsov, The accuracy of certain approximate methods for the computation of weak solutions of a first order quasilinear equation, Ž. Vyčisl. Mat. i Mat. Fiz., 16 (1976), pp. 1489-1502, 1627. English translation: U.S.S.R. Computational Math. and Math. Phys. 16 (1976), no. 6, 105-119 (1978). 
[24] I. MryaderA, Nonlinear Semigroups, vol. 109 of Translations of Mathematical Monographs, A.M.S., 1992.

[25] R. H. Nochetto, A posteriori error control for FBP, Free Boundary Problems: Theory and Applications, eds. P. Colli et al., Birkhäuser, 147 (2004), pp. 207-223.

[26] R. H. Nochetto, G. Savaré, and C. Verdi, Error control for nonlinear evolution equations, C.R. Acad. Sci. Paris, Serie I, 326 (1998), pp. 14371442.

$[27] \_$, A posteriori error estimates for variable time-step discretizations of nonlinear evolution equations, Comm. Pure Appl. Math., 53 (2000), pp. 525-589.

[28] J. Rulla, Error analysis for implicit approximations to solutions to Cauchy problems, SIAM J. Numer. Anal., 33 (1996), pp. 68-87.

[29] G. SAVARÉ, Optimal error estimates for dissipative evolution problems, Free Boundary Problems: Theory and Applications, eds. P. Colli et al., Birkhäuser, 147 (2004), pp. 281-291.

[30] R. E. Showalter, Monotone Operators in Banach Space and Nonlinear Partial Differential Equations, American Mathematical Society, Providence, RI, 1997.

[31] A. Visintin, Differential Models of Hysteresis, Springer-Verlag, Berlin, 1994. 